\title{
A SIMULATION OF THE INTRACLUSTER MEDIUM WITH FEEDBACK FROM CLUSTER GALAXIES
}

\author{
Christopher A. Metzler and August E. Evrard \\ Department of Physics, University of Michigan, Ann Arbor, MI 48109 \\ Received 1993 October 6; accepted 1994 June 29
}

\begin{abstract}
We detail method and report first results from a three-dimensional hydrodynamical and $N$-body simulation of the formation and evolution of a Coma-sized cluster of galaxies, with the intent of studying the history of the hot, X-ray emitting intracluster medium. Cluster gas, galaxies, and dark matter are included in the model. The galaxies and dark matter feel gravitational forces; the cluster gas also undergoes hydrodynamical effects such as shock heating and $P d V$ work. For the first time in three dimensions, we include modeling of ejection of processed gas from the simulated galaxies by winds, including heating and heavy element enrichment. For comparison, we employ a "pure infall" simulation using the same initial conditions but with no galaxies or winds.

We employ an extreme ejection history for galactic feedback in order to define the boundary of likely models. As expected, feedback raises the entropy of the intracluster gas, preventing it from collapsing to densities as high as those attained in the infall model. The effect is more pronounced in subclusters formed at high redshift. The cluster with feedback is always less X-ray luminous, but experiences more rapid luminosity evolution, than the pure infall cluster.

Even employing an extreme ejection model, the final gas temperature is only $\sim 15 \%$ larger than in the infall model. The radial temperature profile is very nearly isothermal within $1.5 \mathrm{Mpc}$. The cluster galaxies in the feedback model have a velocity dispersion $\sim 15 \%$ lower than the dark matter. This results in the true ratio of specific energies in galaxies to gas being less than one, $\beta_{\text {spec }} \sim 0.7$. The infall model predicts $\beta_{\text {spec }} \sim 1.2$. Large excursions in these values occur over time, following the complex dynamical history of the cluster.

The morphology of the X-ray emission is little affected by feedback. The emission profiles of both clusters are well described by the standard $\beta$-model with $\beta_{\mathrm{fit}} \simeq 0.7-0.9$. X-ray mass estimates based on the assumptions of hydrostatic equilibrium and the applicability of the $\beta$-model are quite accurate in both cases.

A strong, radial iron abundance gradient is present, which develops as a consequence of the steepening of the galaxy density profile over time. Spectroscopic observations using nonimaging detectors with wide $\left(\sim 45^{\prime}\right)$ fields of view dramatically smear the gradient. Observations with arcminute resolution, made available with the $A S C A$ satellite, would readily resolve the gradient.
\end{abstract}

Subject headings: cosmology: theory - galaxies: clustering — galaxies: X-rays - intergalactic medium

\section{INTRODUCTION}

The space between galaxies in clusters is filled with a hot, $\mathrm{X}$-ray emitting plasma known as the intracluster medium, or ICM. The study of the origin and evolution of this gas is guided by three observations. The ratio of the mass in the ICM to the mass in the cluster galaxies, $M_{\mathrm{ICM}} / M_{\text {stars }}$, seems to increase from $\sim 1$ for poorer clusters to $\sim 4-6$ for rich clusters (cf. David et al. 1990a); that this ratio is greater than one implies that the majority of the gas has not been processed through cluster galaxies. On the other hand, spectroscopic observation of clusters show iron abundances in the ICM of about one-third solar, implying that at least some of the ICM has been cycled through stars. Finally, the ratio of specific energy in cluster galaxies to specific energy in cluster gas, the quantity $\beta$ discussed below, seems to be less than one for a majority of systems (cf. Edge \& Stewart 1991), implying more energy per unit mass in gas than in galaxies. Any good theory of the origin and evolution of the ICM must account for these observations.

The paradigm for the formation of clusters and the ICM involves the infall of galaxies and gas into a hierarchically developing potential well (Gunn \& Gott 1972). The combination of gravitational collapse and shock heating drives the gas up to observed temperatures $\left(T \sim 10^{7}-10^{8} \mathrm{~K}\right)$. Such a scenario can account for the huge amount of gas in clusters, but cannot immediately account for the presence of metals in the gas. There are three sources for the processed gas in the ICM commonly considered: (1) a "background" metallicity in the infalling gas, perhaps from Population III stars (Carr, Bond, \& Arnett 1984); (2) processed gas ram pressure stripped out of cluster galaxies by the existing ICM (Gunn \& Gott 1972; Biermann 1978; Takeda, Nulsen, \& Fabian 1984; Gaetz, Salpeter, \& Shaviv 1987); or (3) processed gas driven out of cluster galaxies by energetic winds.

If cluster metallicities originate predominantly from abundances in the gas that existed before the cluster formed, then, neglecting settling of heavy ions, one would expect no radial gradient in metallicity in the cluster. One would also expect cluster metal abundances to be independent of the mass of the cluster. While observed iron abundances in rich clusters lie within a fairly narrow range, there does exist some evidence for a decrease in metal abundance with cluster mass (cf. Jones \& Forman 1992). If the metals come from processed gas that has been stripped out of cluster galaxies, then one would expect a strong peak in metallicity near the center, where the density of the ICM is the largest. 
Ejection by galactic winds was originally considered as a source for the ICM (Yahil \& Ostriker 1973; Larson \& Dinerstein 1975). As a galaxy evolves, supernova activity affects the state of the interstellar medium. Subsonic galactic outflows or transonic galactic winds can develop, which in turn eject hot, processed gas out of the galaxy. Winds have been observed in starburst galaxies (cf. McCarthy, Heckman, \& van Bruegel 1987; Heckman, Armus, \& Miley 1987, 1990), and winds and outflows are general properties of models of the evolution of ellipticals (Matteucci \& Tornambè 1987; Matteucci \& Vettolani 1988; David, Forman, \& Jones 1990b, 1991b; Ciotti et al. 1991). It seems reasonable to assume that some feedback of processed gas from cluster galaxies into the ICM would take place. A distinguishing characteristic of the wind scenario is that it results in not only metals, but also energy, being injected into the ICM. The typical specific energies associated with supernova-driven galactic winds are on the order of, or greater than, the specific energies associated with infall into a rich cluster, possibly resulting in greater specific energy for cluster gas than one finds for cluster galaxies. Winds thus provide a simple mechanism for explaining $\beta<1$.

Simulations are necessary to accurately model cluster evolution and test these hypotheses. In the 1970 s, various papers studied the formation and evolution of an X-ray cluster (e.g., Gull \& Northover 1975; Lea 1976; Takahara et al. 1976; Cowie \& Perrenod 1978; Perrenod 1978). The simulations associated with these papers were one-dimensional hydrodynamical models, usually with fixed, static graviational potentials. Perrenod (1978) introduced a time-varying potential into his one-dimensional simulations which was extracted from the $N$-body simulation of White (1976). Cowie \& Perrenod (1978) and Perrenod (1978) also considered models in which the entire ICM originated from within cluster galaxies, assuming some static, or simply varying in space and time, mass ejection rate $\phi(r, t)$. Evrard $(1990 \mathrm{a}, \mathrm{b})$ performed three-dimensional simulations of X-ray clusters where, rather than adopting some ad hoc gravitational potential, both collisionless dark matter and collisional baryonic fluids were included; the baryons fell into the developing dark matter potential well and underwent shock heating. Thomas \& Couchman (1992), Katz \& White (1993), and Tsai, Katz, \& Bertschinger (1994) also studied similar three-dimensional models with somewhat higher numerical resolution. This paper extends the work of Evrard (1990b), improving resolution and allowing examination of the effects of galactic winds upon the intracluster medium. In particular, we wish to compare the evolution of a simulated cluster with and without energetic ejection from galaxies, to isolate any changes originating from the additional physics of galactic feedback. Since this work is intended to be exploratory, we take an extreme model for the strength of such winds, with the intention of defining the "upper envelope" of effects on cluster evolution. Future papers will address plausible models for such energetic ejection, contrast winds with gas-dynamical stripping, and use of an ensemble of simulated clusters to explore correlations of observables as well as X-ray and optical indicators of dynamical state.

This paper is organized as follows. Section 2 describes the numerical method used in modelling feedback from galaxies. The specific cluster model studied for this paper, and the results of the simulations, are described in $\S 3$. Section 4 considers what these simulations can tell us about cluster mass estimates, the cluster baryon fraction and the specific energies of various cluster mass components. Finally, $\S 5$ summarizes the results, lists our conclusions, and presents ideas for further study.

\section{METHOD}

\subsection{Cosmological Model, Initial Conditions, and Simulation Algorithm}

The parameters for our models follow those of the standard biased cold dark matter (CDM) scenario (Blumenthal et al. 1984; Davis et al. 1985): $\Omega=1$, baryonic fraction $\Omega_{b}=0.1$, Hubble constant $h=0.5$ (where $h \equiv H_{0} / 100 \mathrm{~km} \mathrm{~s}^{-1} \mathrm{Mpc}^{-1}$ ) and the spectrum is normalized to have a linear evolved variance in $16 \mathrm{Mpc}$ spheres of $\sigma_{8}=0.59$, equivalent to a bias factor $b \equiv \sigma_{8}^{-1}=1.7$. An initial density field is generated in a $40 \mathrm{Mpc}$ cube from a CDM power spectrum using the path integral method of Bertschinger (1987), which constrains the field smoothed with a Gaussian filter to have a specified value at the center of the simulated volume. For this model, we smooth over a length scale of $R_{f}=8 \mathrm{Mpc}$, corresponding to a mass scale of $M_{f}=(2 \pi)^{3 / 2} R_{f}^{3} \rho=5.6 \times 10^{14} M_{\odot}$ (Bardeen et al. 1986). The perturbation height at the center was constrained to a value $\delta_{c}=2.0$ when filtered on scale $M_{f}$, resulting in a $3.6 \sigma$ peak on this scale near the center of the box. The primordial density field is used to generate a particle distribution at the starting redshift using the Zel'dovich approximation, as described in Efstathiou et al. (1985).

Our simulation algorithm P3MSPH allows us to track the evolution of both collisionless dark matter and collisional baryonic fluids. The particles of all fluids are coupled together by gravity; the baryonic fluid also feels gas forces. The effects of gravity are modeled by the well-known particle-particleparticle-mesh $\left(P^{3} M\right)$ algorithm of Efstathiou \& Eastwood (1981); a detailed explanation is in Hockney \& Eastwood (1981). The hydrodynamical terms in the force equations are solved using the smoothed particle hydrodynamics (SPH) algorithm of Gingold \& Monaghan (1977). A review of SPH can be found in Monaghan (1992). The combined algorithm is described in Evrard (1988) and a description of some of the analysis techniques used here is provided in Evrard (1990b).

\subsection{Inclusion of Galaxies and Ejection of Gas}

Previous efforts to model the effects on the ICM of ejection of gas from galaxies were limited by their assumed spherical symmetry (e.g., Cowie \& Perrenod 1978; Perrenod 1978). Mass and energy ejection was typically parameterized by some ad hoc mass injection rate $\phi(r, t), r$ being the radial distance from the center of the cluster. The three-dimensional capability of our simulation code allows us to consider time-dependent mass ejection rates which truly vary with position. Since cluster galaxies are the source of this processed gas, the time evolution of the three-dimensional mass ejection rate $\phi(r, t)$ depends on both the evolution of the spatial distribution of galaxies and the evolution of the galaxies themselves. Therefore, in addition to simulating background fluids of collisionless dark matter and collisional baryonic gas, we also simulate a third set of particles representing individual cluster galaxies. The galaxy phase-space distribution evolves consistently with the other two fluids, being coupled to them by gravity. This method offers another benefit; by modeling the galaxy motions explicitly, we can study the dynamics of the ensemble of galaxies as well as the evolution of the ICM. Analysis of cluster galaxy kinematics can be done without having to make the assumption that the evolution of the galaxy distribution is well approximated by the evolution of the dark matter distribution. 
In particular, issues of velocity bias, anisotropies in galaxy velocity dispersions, and optical substructure can be addressed.

Ideally, our simulation would include the formation of individual galaxies, which would then be the source of ejected material. However, simulations which can model galaxy formation in such a cosmological context require a large amount of computational overhead. Less numerically ambitious simulations will allow us to generate many runs and perform statistical analyses, but we consequently cannot adequately resolve scales relevant for galaxy formation. We therefore approximate what we cannot resolve by putting galaxies in 'by hand', at locations corresponding to peaks in the initial density field. The correspondence between peaks and subsequent collapsed objects has been investigated in $N$-body experiments by Frenk et al. (1988) and Katz, Quinn, \& Gelb (1992). These studies have shown that high peaks are very likely to pick out sites which later collapse into bound structures, but the correspondence is very poor for low peaks. We thus limit our analysis to high peaks in an attempt to approximate the behavior of $\mathrm{L}^{*}$ galaxies. It should be kept in mind that this is an approximate treatment, intended to generate the observed number density of bright galaxies in a manner consistent with their statistical clustering properties (Davis et al. 1985). While the method is certainly artificial, it is not entirely without basis. Evrard, Silk, \& Szalay (1990), for instance, found that an identification of peaks with galaxies, combined with a simple mapping between peak heights and galaxy morphology, could reproduce the observed morphology-density relation (e.g. Dressler 1980).

To locate sites for galaxy insertion, we filter the initial density field on a scale appropriate for massive, $L^{*}$ galaxies. We use Gaussian filtering with $R_{f}=0.5 \mathrm{Mpc}$ and identify sites above a threshold of $2.5 \sigma$ as locations for massive galaxy formation (Davis et al. 1985). We then return to the initial particle distribution and replace the gas particles associated with each peak with a composite "galaxy particle." The initial linear momentum of a galaxy particle is set by demanding conservation of linear momentum when the gas particles are removed. Applying this technique yielded 108 " $\mathrm{L}^{*}$ galaxies," with an average mass of $5.06 \times 10^{11} M_{\odot}$. The number density, for a comoving $40 \mathrm{Mpc}$ box, is then $1.69 \times 10^{-3} \mathrm{Mpc}^{-3}$, very similar to the most recent estimate of the number density of bright galaxies in the Stromlo-APM survey $\phi^{*}=1.75 \times 10^{-3}$ $\mathrm{Mpc}^{-3}$ (Loveday et al. 1992).

The modeling of galactic winds requires several approximations. The formation, duration, intensity, and composition of such winds are highly dependent upon the assumed stellar initial mass function (IMF) and star formation rate (SFR), as these determine stellar mass loss rates, as well as rates of Types I and II supernovae. For instance, David et al. (1990b, 1991a) and Ciotti et al. (1991) used hydrodynamical simulations to explore a variety of models of elliptical galaxies with different IMFs and SFRs; the wind solutions obtained had similar general properties, but varied strongly in details. There is no "most general case" appropriate for our use. We have thus coded a general method capable of handling a variety of possible wind models. To parameterize the behavior of the individual galaxies, the simulation code take time-dependent specific mass, energy, and iron mass ejection rates as input. Such rate curves in reality depend in a complicated fashion on the state of the interstellar medium. The simple curves implemented in this work will be discussed in the next section.
For each galaxy, as a model advances in timesteps, the specific mass ejection rate curve is integrated in time until the ejected mass is equal to the mass of a gas particle. At that point, a new gas particle is created at the location of the galaxy particle, and the mass of the galaxy particle is decremented by one. The energy and iron mass fraction of the new gas particle are determined by integrating those curves over the same time period.

There are three naive concerns with this algorithm. It is unclear from galaxy evolutionary models what fraction of the energy of the wind should be in mechanical energy and what should be in thermal energy. Second, an actual galactic outflow ejects a continuous stream of matter which interacts with the large surrounding volume of gas. In contrast, the discrete particle and discrete timestep nature of our simulations necessitates that lumps of gas (individual gas particles) be ejected periodically, with a large total energy. We must ensure that the global properties of the ejected matter, and its effects upon the rest of the ICM, are not strongly sensitive to the discreteness limits of our ejection procedure. Finally, the SPH algorithm is inaccurate and unstable when hydrodynamic properties vary strongly on the scale of one particle, as could happen in a poorly modeled ejection process.

All three of these issues are resolved by addressing the second. Modeling a continuous flow as a discrete particle neglects the interaction with the surrounding medium that would have taken place in the time up to when the particle is ejected. We therefore adopt a paradigm for what happens to ejected gas, and force such interaction to take place at the time of particle ejection. We mimic the mixing which takes place on unresolved scales by smoothing the ejected energy and momentum with the $N$ nearest particles of the ejected particle. We use $N=30$, although tests showed that the characteristics of runs on resolved scales were insensitive to varying $N$ between 10 and 30. During the runs, we continuously compared the distance over which we forced interaction with the sound speed in the ejected gas, to verify that the interactions we have inserted by hand do not violate causality bounds.

The paradigm for how the wind-ICM interaction is forced is as follows. In the rest frame of a galaxy, we assume its outflow to be isotropic; then no momentum is associated with that outflow, and we can define a net momentum of our interacting system to be just the sum of the momenta of the $N$ neighbors:

$$
\boldsymbol{p}_{\mathrm{tot}}=\sum_{i=1}^{N} \boldsymbol{p}_{i}
$$

Upon meeting the surrounding medium, the ejected gas particle should gain momentum from its neighbors; but the total momentum of the ejected particle plus neighbors, $\boldsymbol{p}_{\text {tot }}$, should be conserved. Then, in the rest frame of the ejecting galaxy, the ejected gas particle's initial momentum is chosen to be the average of the resulting flow:

$$
\boldsymbol{p}_{\mathrm{ej}}=\frac{\boldsymbol{p}_{\mathrm{tot}}}{\mathrm{N}+1}
$$

To bring the ejected particle up to this momentum (and to conserve net momentum), each neighbor is assumed to have lost $1 / N$ the momentum given to the ejected particle:

$$
\boldsymbol{p}_{i}^{\prime}=\boldsymbol{p}_{i}-\frac{\boldsymbol{p}_{\mathrm{ej}}}{N}
$$


Such a process conserves momentum, but it does not conserve energy. A brief calculation shows that an amount of kinetic energy is lost equal to

$$
E_{\text {lost }}=\frac{\left\|\boldsymbol{p}_{\text {tot }}\right\|^{2}}{2 m N(N+1)}
$$

with $m$ the mass of a gas particle. But this kinetic energy loss is not a problem. In the galaxy's frame, as the ejected gas encounters the local flow of the intracluster medium and momentum is transferred, one expects kinetic energy to be converted into heat; this is an inelastic process. The energy $E_{\text {lost }}$ above is thus added to the thermal energy we obtained by integrating the wind luminosity curve; this is the total thermal energy available from the ejection process. To complete the consideration of the interaction of the wind with its surroundings, the thermal energies of the ejected particle and the $N$ neighbors are adjusted to the same, average value of the total thermal energy available divided by $N+1$; in effect, the ejected particle is created in thermal equilibrium with its surroundings.

Globally, energy is not conserved, as the energy associated with the new gas particle supposedly came from nuclear processes within the galaxy, and such sources of energy are not considered in summing the total kinetic, potential, and thermal energy in the simulation volume. However, except for the "new energy" associated with the wind luminosity, all existing energy and momentum is conserved in this interaction process.

Because energy and momentum are mixed between the ejected matter and the surrounding gas, the iron in the wind must be mixed as well. We assume that the mixing works to drive the iron mass fraction in ejected particles toward the average with its surrounding $N$ neighbors. We thus write the iron fraction of the ejected particle after mixing, $f_{\mathrm{ej}}^{\prime}$ as

$$
f_{\mathrm{ej}}^{\prime}=\bar{f}+k\left(f_{\mathrm{ej}}-\bar{f}\right),
$$

where $f_{\mathrm{ej}}$ is the value before mixing, $\bar{f}$ is the average, and $\mathrm{k}$ is number from zero to one which controls the strength of the mixing. If $k=1$, the iron fraction for the ejected particle doesn't change; if $k=0, f_{\mathrm{ej}}^{\prime}=\bar{f}$. The change in neighbor $j$ 's iron fraction is determined by a conservation argument to be

$$
f_{j}^{\prime}=f_{j}+\frac{(1-k)}{N}\left(f_{\mathrm{ej}}-\bar{f}\right)
$$

In discussing the iron distribution, our standard run assumes strong mixing $(k=1 / 30)$, but we will also present results for a run conducted with no mixing $(k=1)$.

It should be noted that there are relevant physical effects which are not included in this work. Our code is equipped to include the effects of radiative cooling; but we have ignored cooling because of our poor resolution on scales where cooling should be important. We expect to include cooling in future runs with higher resolution. We also do not include gasdynamical stripping from cluster galaxies (Gunn \& Gott 1972; Biermann 1978; Takeda et al. 1984; Gaetz et al. 1987); this should be an important effect, and a future paper will compare stripping with energetic ejection and contrast their effects on the thermal state of, and metallicity distribution in, the ICM. Several other relevant physical processes have been neglected: the heating of the ICM by galaxy motions and friction (Rephaeli \& Salpeter 1980; Just et al. 1990); heating by relativistic electrons from radio galaxies (Lea \& Holman 1978; Rephaeli 1979); and thermal and mass transport by electron conduction (Rephaeli 1977; David, Hughes, \& Tucker 1992a). We neglect these effects because they are considered weak relative to the physics currently in place in our simulation algorithm.

\section{MODELS}

\subsection{Description of Models}

A main purpose of this work is to highlight the differences in cluster evolution associated with including feedback from galaxies. Therefore, in addition to the simulation of galaxies, gas, and dark matter, we also evolved the same initial conditions as a two-fluid system of gas and dark matter only. The version with galaxies then also included ejection of mass and energy from those galaxies. In the figures and tables below, we refer to the run with ejection using the label EJ, while the run without ejection will be labeled $2 \mathrm{~F}$, for two-fluid.

To further make clear the direction in which physical properties change when feedback is included, we considered a simple and extreme set of ejection curves. We assumed that galaxies eject mass and energy at a flat, constant rate from a redshift $z=4.0$ to the present. The amplitude of this rate was set by requiring that over this ejection period, the simulated galaxies blow off half their mass - the most extreme scenario considered in simulations such as David et al. (1991b) and analytical arguments such as White (1991). In setting the wind luminosity, we took a flat value for a model $10^{10} \mathrm{~L}_{\odot}$ galaxy and scaled all other galaxies linearly from this value, assuming a mass to light ratio of 8 . This yields a wind luminosity $L_{\text {wind }}=4 \times 10^{42}$ ergs s$^{-1}$ for a $10^{10} L_{\odot}$ galaxy.

Realistic models and recent ASCA observations (Mushotzky 1994) indicate that most of the energy and mass is ejected early in a phase dominated by Type II SN. Flattening this peak out over a Hubble time leads to a large overestimate of the present expected wind luminosity of ellipticals; our value is about a factor of 50 times larger than the current estimated Type Ia supernova output in ellipticals. The overall energetics are on the extreme edge. Renzini et al. (1993) argues that a $10^{10} L_{\odot}$ galaxy produces $\sim 10^{60}$ ergs of energy from supernovae over its lifetime. Averaged over a Hubble time, this produces a luminosity on the order of the value above. However, this is still an overestimate by perhaps an order of magnitude, because $\sim 90 \%$ of the energy may be lost to radiation.

Still, we use the above $L_{\text {wind }}$ in the spirit of studying an extreme model in order to define the outer boundary of the effects on cluster evolution. To explore how this assumption affects our results, we have also performed a run, using otherwise identical conditions, in which the winds last for only $26 \%$ of a Hubble time. The galaxies in this run generate roughly one-fourth less total energy, mass, and metals than our standard EJ case. This history is closer to the expectations of mainstream wind models. As we will see below, the differences between the two ejection models are not dramatic.

The parameters of the EJ run, and the properties of the specific set of initial conditions used, are summarized in Table 1.

\subsection{Model Evolution}

Figure 1 shows the evolution of a slice through the simulation volume at six redshifts. The four columns show the distribution of dark matter, all gas, ejected gas, and galaxies in the slice; only one-seventh of the dark matter and gas particles in the slice are shown for clarity. At early times, the filamentary distribution of matter in the volume is evident. As the run 


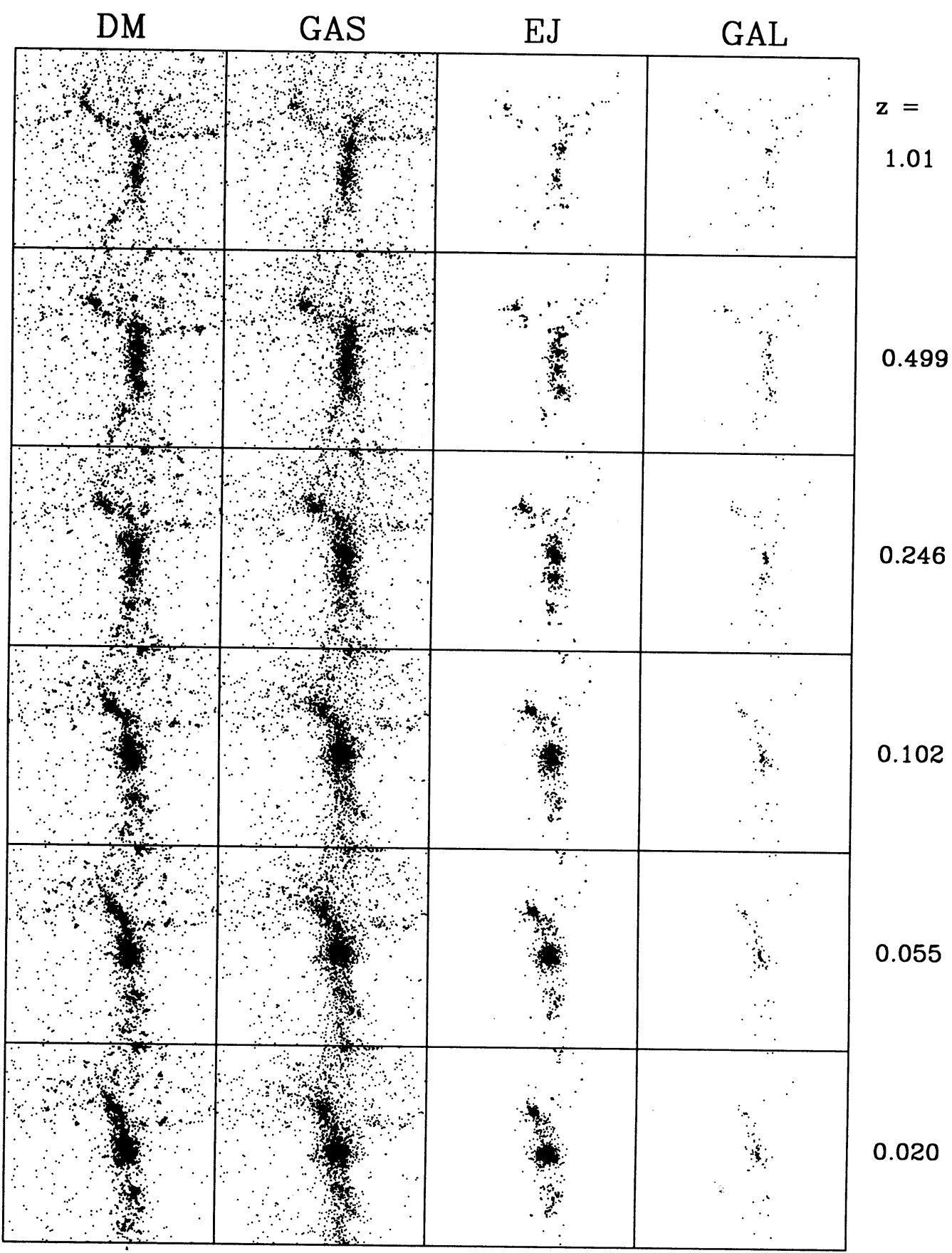

FIG. 1.-Evolution of the fluids in the simulation. Each frame shows the particles contained within a slice of comoving dimensions $33.5 \mathrm{Mpc}$ by $33.5 \mathrm{Mpc}$ wide, $6.7 \mathrm{Mpc}$ thick, projected along the $z$-axis. Each row corresponds to a given redshift, which is shown to the right of the panels. The first two columns show the dark matter and gas distributions, sampling $1 / 7$ of the particles for clarity. The third column shows the distribution of all ejected gas particles in the slice; it is thus a subset of the full (not sparsely sampled) gas distribution. The fourth column shows the location of the galaxy particles within the slice.

progresses, matter drains via these filaments into subclusters, which then collect to form the final cluster. In the figure, protocluster potential wells clearly have formed by a redshift of 1 . Four separate subclusters are in place at a redshift of a half; these undergo mergers by $z=0.1$. Some elongation from the merger sequence persists by $z=0.02$, while the gas has adopted a more rounded distribution since $z=0.1$. A smaller group of galaxies, with associated gas and dark matter, is clear to the north and slightly east of the main cluster, $\sim 7 \mathrm{Mpc}$ away.

\subsection{Temperature, Density, and Metallicity Profiles}

The evolution of the temperature profile for the cluster is shown in Figure 2, for both the ejection (EJ) and two-fluid (2F) runs. Because there exists several comparable subclusters before $z=0.246$, we define the "cluster" as the most massive of these in displaying these profiles. In both cases, the cluster relaxes to a roughly isothermal distribution within $1-2 \mathrm{Mpc}$ at $z=0$. The inner temperature profile is more nearly level for the ejection run, though the difference is small. The temperature 
TABLE 1

Summary OF Model Parameters

\begin{tabular}{|c|c|}
\hline Parameter & Value \\
\hline Comoving box length (Mpc) & 40 \\
\hline Total $\mathrm{n}$ & $4.44 \times 10^{15}$ \\
\hline Number of dark matter particles & 32768 \\
\hline Mass per dark matter particle $\left(M_{\odot}\right)$. & $1.22 \times 10^{11}$ \\
\hline Initial number of gas particles & 28733 \\
\hline Mass per gas particle $\left(M_{\odot}\right)$. & $1.35 \times 10^{10}$ \\
\hline Number of "galaxies". & 108 \\
\hline Comoving gravitational sc & 78 \\
\hline Minimum smoothing length $h_{\min }(\mathrm{kpc}) \ldots \ldots \ldots$ & 110 \\
\hline Initial redshift .... & 9 \\
\hline Initial mean overdensity .. & 0.031 \\
\hline Timestep $(\mathrm{yr}) \ldots \ldots \ldots \ldots \ldots$ & $1.33 \times 10^{7}$ \\
\hline Initial temperature of gas & $10^{4} \mathrm{~K}$ \\
\hline Specific energy of $w$ & $1.37 \times 10^{8} \mathrm{~K}$ \\
\hline
\end{tabular}

decreases radially as $T \propto r^{-0.88}$ from $\sim 2 \mathrm{Mpc}$ out to $\sim 7$ $\mathrm{Mpc}$, where it briefly rises because of the subcluster at that distance (visible in Fig. 1). The isothermal region within $2 \mathrm{Mpc}$ contains a total mass of $9.4 \times 10^{14} M_{\odot}$, corresponding to a mean interior density $\sim 400$ times the mean background value.

The standard descriptive model for the distribution of matter in clusters is the so-called hydrostatic isothermal $\beta$-model (Cavaliere \& Fusco-Femiano 1976, 1978; Sarazin \& Bahcall 1977). This model presumes that fluids making up the cluster are distributed with a radial density function that can be written as

$$
\rho=\rho_{0}\left[1+\left(\frac{r}{r_{c}}\right)^{2}\right]^{-3 \alpha / 2}
$$

where $\rho_{0}$ is the central density and $r_{c}$ is the "core radius." The three parameters $\rho_{0}, r_{c}$, and $\alpha$ have values that are peculiar to each fluid. In the simplest model, the exponent $\alpha$ for the gas density profile is equal to $\beta$, defined as the ratio of specific energies in galaxies and gas,

$$
\beta=\frac{\sigma_{r}^{2}}{\left(k T / \mu m_{p}\right)} .
$$

Here $\sigma_{r}$ is the line-of-sight velocity dispersion of galaxies in the cluster, $T$ is the gas temperature, $\mu$ the mean molecular weight and $m_{p}$ the proton mass. Beyond the hydrostatic equilibrium assumption, a number of other assumptions are involved in relating $\alpha_{\mathrm{ICM}}$ to the ratio of specific energies: (1) the galaxies and binding mass must have density profiles obeying equation (7) with $\alpha=1$; (2) galaxy orbits are isotropic; and (3) the gas is supported purely by thermal pressure. Because each of these assumptions are unlikely to be true to $\sim 20 \%$ accuracy, the validity of the simple model is questionable. In particular, the use of $\beta$ values measured from X-ray surface brightness profiles as an estimate of the ratio of specific energies is prone to systematic error (Evrard 1990b; but see Thomas and Couchman 1992 for an alternative view). Still, we can take equation (7) in the spirit of a fitting function and then examine the correspon-

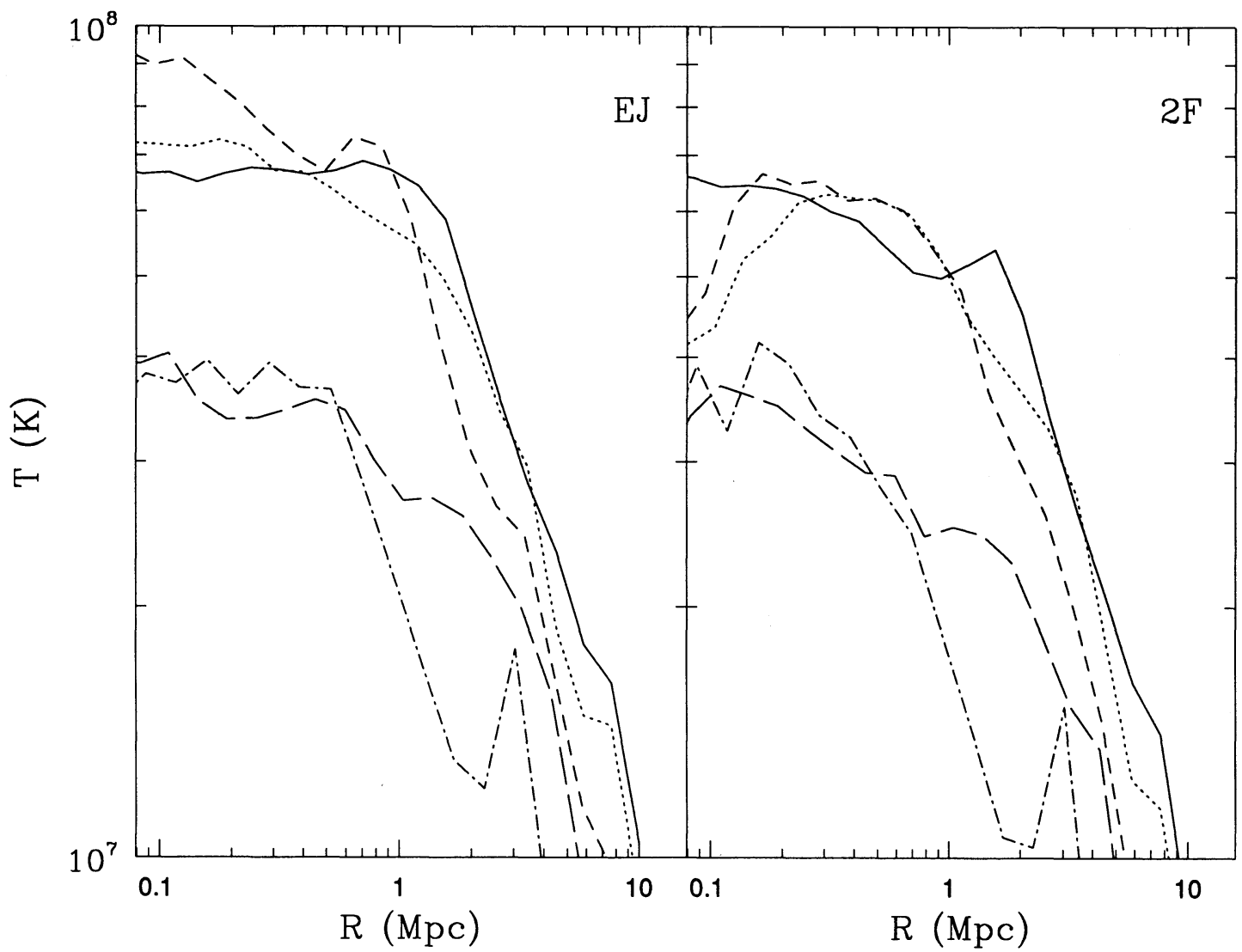

FIG. 2.-Temperature profile of the cluster gas for the ejection (EJ) and two-fluid (2F) runs. The profiles are centered on the potential minimum of the cluster, with the cluster at any epoch defined as the most massive progenitor of the final cluster. The five lines correspond to different redshifts: solid, $z=0.02 ;$ dotted, $z=0.102$; short-dashed, $z=0.246$; long-dashed, $z=0.499$; and dash-dot, $z=1.01$. 
in dence between the values of $\alpha$ obtained when fitting density profiles and the ratio of specific energies. This will be discussed $\therefore$ in the following section.

We fitted this model to the actual dark matter and gas radial density profiles using the average density in concentric spherInI ical shells centered on the most bound dark matter particle in the cluster (effectively, the bottom of the potential well). The top row of Figure 3 shows the dark matter density profile, at $z=0.02$, for the ejection and two-fluid runs. The lines correspond to best-fits for different values of the cutoff radius - the maximum radius of data to use in the fit; Table 2 shows the best-fit parameters. The dark matter displays very similar

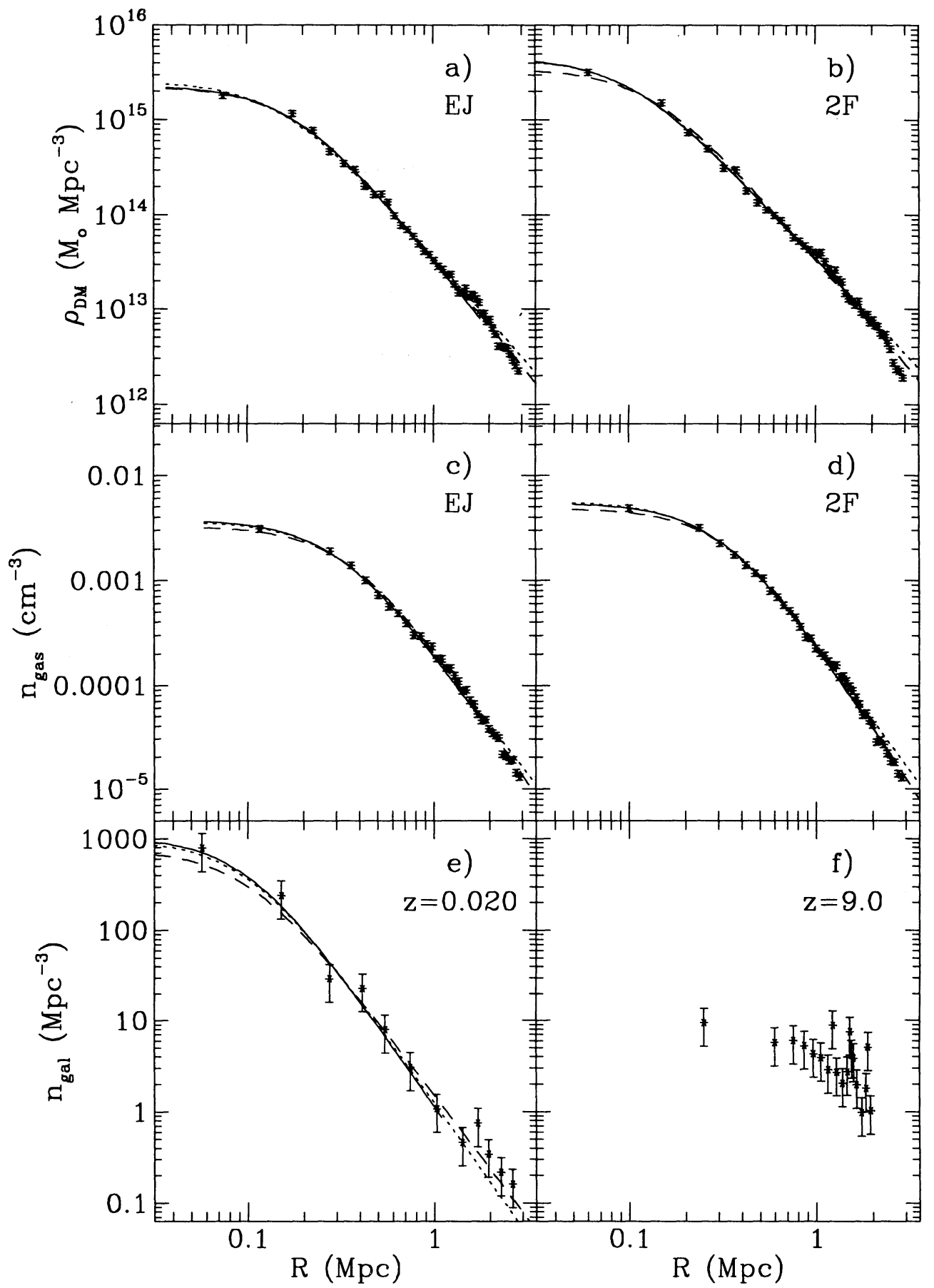

FIG. 3.-Density profiles of the dark matter, gas and galaxies. Data bins are Lagrangian; they are defined by 200 particles per bin for the dark matter and gas, and by 5 particles per bin for the galaxies. (a) and (b) Least-squares best-fits of the true dark matter density profile of the cluster, at $z=0.02$, to eq. (7). Left plot is from the ejection run; right plot is from the two-fluid run. The smallest radial point was not used in calculating the fit. Three lines are present in each plot, corresponding to fits where data points beyond $1 \mathrm{Mpc}$ (solid curve), $2 \mathrm{Mpc}$ (dotted curve), and $3 \mathrm{Mpc}$ (dashed curve) were excluded from the fit. The primary difference between the three lies in the large radius slope (value of $\left.\alpha_{D M}\right) .(c)$ and $(d)$ Least-squares best fits of the true gas number density profile of the cluster at $z=0.02$, to eq. (7). Data plotted as for the dark matter, in $(a)$ and $(b)$. $(e)$ and $(f)$ Galaxy number density profile of the cluster at $z=0.02(e)$ and at $z=9.0(f)$. Least-squares best fits of the $z=0.02$ data to eq. (7) are shown, using the same fitting constraints as in $(a)-(d)$ above. The "center" of the cluster at high redshift is determined by identifying the dark matter making up the cluster at $z=0.02$, and using the center of that set of particles' distribution at $z=9.0$. 
TABLE 2

Dark Matter Density Profile Fits

\begin{tabular}{cccccc}
\hline \hline Run & $\begin{array}{c}\text { Cutoff Radius } \\
(\mathrm{Mpc})\end{array}$ & $\left(M_{\odot} \rho_{\mathrm{Mpc}}{ }^{-3}\right)$ & $\begin{array}{c}r_{c} \\
(\mathrm{kpc})\end{array}$ & $\alpha_{\mathrm{DM}}$ & $\chi^{2} /$ d.o.f. \\
\hline EJ $\ldots \ldots$ & 1.0 & $(2.30 \pm 0.19) \times 10^{15}$ & $180 \pm 14$ & $0.823 \pm 0.032$ & 1.59 \\
& 2.0 & $(2.53 \pm 0.20) \times 10^{15}$ & $151 \pm 9$ & $0.748 \pm 0.011$ & 2.07 \\
& 3.0 & $(2.24 \pm 0.16) \times 10^{15}$ & $179 \pm 8$ & $0.804 \pm 0.008$ & 3.06 \\
$2 \mathrm{~F} \ldots \ldots$ & 1.0 & $(4.57 \pm 0.49) \times 10^{15}$ & $100 \pm 9$ & $0.709 \pm 0.021$ & 1.51 \\
& 2.0 & $(4.47 \pm 0.43) \times 10^{15}$ & $102 \pm 7$ & $0.710 \pm 0.009$ & 2.44 \\
& 3.0 & $(3.48 \pm 0.30) \times 10^{15}$ & $135 \pm 7$ & $0.772 \pm 0.007$ & 5.42 \\
\hline
\end{tabular}

forms in the $\mathrm{EJ}$ and $2 \mathrm{~F}$ runs, as naively expected. The core radii for the EJ fits are not much more than our resolution limit; there is no evidence for a core to the dark matter distribution, in agreement with Dubinski \& Carlberg (1991), who examined a halo of smaller mass with higher resolution. Indeed, the use of equation (7) to model the dark matter is not well motiviated, since there is no obvious physics operating on the dark matter from which one could attempt to derive a core radius (Crone, Evrard, \& Richstone 1994).

The central density of the dark matter in the EJ run is slightly lower than that of the $2 \mathrm{~F}$ run, and the core radii and $\alpha_{\mathrm{DM}}$ value larger, respectively. This may be expected if dynamical friction were transferring energy from the galaxies to the dark matter in the EJ run (recall there are no galaxies in the $2 \mathrm{~F}$ model). Within $\sim 200 \mathrm{kpc}$, the timescale for orbital decay due to dynamical friction (Binney \& Tremaine 1987) is $\sim 1 \mathrm{Gyr}$, so it is not implausible that the dark matter may be affected in the central regions. However, the effect is weak; a second fit to the $2 \mathrm{~F}$ run, with a $1.0 \mathrm{Mpc}$ cutoff and a fixed $180 \mathrm{kpc}$ core radius (the same as the EJ run for a $1.0 \mathrm{Mpc}$ cutoff), produced values for the central density and $\alpha_{\mathrm{DM}}$ equivalent to those for the EJ run. Furthermore, the two innermost data points for both runs, which seen to show the difference in central density, lie below or near our resolution limit; our resolution is not sufficient to make a defensible statement on the matter.

It is also worthwhile to note the dependence of the best-fit parameters upon the cutoff radius. The dispersion of the data points at larger radii seems to increase, and there are regions where the local slope seems to be steeper or shallower than neighboring regions. This behavior can be expected simply because of the elongated shape of the dark matter, evident in Figure 1. The best-fit value of $\alpha_{D M}$ thus is strongly influenced by the choice of cutoff radius. It is thus inappropriate to consider the dark matter density profile of this cluster at large radii to be a simple power law, $\rho \propto r^{-3 \alpha_{\mathrm{DM}}}$; the logarithmic slope changes with radius.

The gas density profiles of the two runs are compared in the middle row of Figure 3. The curves shown are fits to equation (5) for the same three values of the fitting cutoff radius; Table 3 shows the best-fit parameters. The fitted value of $\alpha_{\text {ICM }}$ shows a dependence on the cutoff radius of the fit, just as the dark matter does. The central gas density in the EJ run is lower than that of the $2 \mathrm{~F}$ run. This difference is due to the energy input from gas ejection. Early on, when the local intergalactic medium is still cold, gas which is blown out of cluster galaxies early drives the surrounding gas onto a higher adiabat. Figure 4 displays the entropy as a function of radius at several redshifts, verifying that the central gas in the EJ run lies on a higher adiabat than the gas in the $2 \mathrm{~F}$ run. The central entropy appears briefly elevated for the $z=0.246$ profile; this is spurious, and results from centering on the bottom of the potential, which is briefly offset from the low-adiabat gas after a merger event. Because the entropy profile of the cluster rises with radius, the preheated gas ends up further from the center of the cluster potential well. The end result is the ICM being "puffed up," to a lower central density than a pure infall scenario. Previously, Kaiser (1991) and Evrard \& Henry (1991) examined analytic models invoking this effect, while Navarro, Frenk, \& White (1993) and Evrard (1990b) have run simulations with gas initially set to a fixed, high entropy.

The corresponding best fit $\alpha_{\mathrm{ICM}}$ is smaller in the EJ case than in the $2 \mathrm{~F}$ run at $z=0.02$, but the effect is only marginally significant, since $\alpha_{\mathrm{ICM}}=0.83 \pm 0.09$ and $0.92 \pm 0.08$ in the EJ and $2 \mathrm{~F}$ models, respectively. Meanwhile, we show below that the value of $\beta$ deduced from surface brightness profiles changes with time as the cluster experiences periods of different dynamical activity (e.g., pre- or postmerger states).

The bottom row of Figure 3 shows the galaxy density profile of the final cluster at $z=9.0$ (the initial distribution of galaxies) and $z=0.02$. The cluster's "initial location" was defined by the center of mass of all the dark matter particles that end up in the cluster at the end of the run. The profile steepens and can be fitted to equation (7) with $\alpha_{\text {gal }}=0.99 \pm 0.35$ at $z=0.02$; the number statistics are poor and the error bars correspondingly

TABLE 3

Gas Density Profile Fits

\begin{tabular}{cccccc}
\hline \hline Run & $\begin{array}{c}\text { Cutoff Radius } \\
(\mathrm{Mpc})\end{array}$ & $\begin{array}{c}n_{0} \\
\left(\mathrm{~cm}^{-3}\right)\end{array}$ & $\begin{array}{c}r_{c} \\
(\mathrm{kpc})\end{array}$ & $\alpha_{\mathrm{ICM}}$ & $\chi^{2} /$ d.o.f. \\
\hline EJ $\ldots \ldots$. & 1.0 & $(3.76 \pm 0.33) \times 10^{-3}$ & $312 \pm 44$ & $0.828 \pm 0.087$ & 0.76 \\
& 2.0 & $(3.65 \pm 0.28) \times 10^{-3}$ & $311 \pm 21$ & $0.796 \pm 0.022$ & 1.38 \\
& 3.0 & $(3.26 \pm 0.22) \times 10^{-3}$ & $365 \pm 18$ & $0.868 \pm 0.015$ & 1.76 \\
$2 \mathrm{~F} \ldots \ldots$. & 1.0 & $(5.44 \pm 0.42) \times 10^{-3}$ & $331 \pm 37$ & $0.915 \pm 0.075$ & 0.35 \\
& 2.0 & $(5.63 \pm 0.38) \times 10^{-3}$ & $300 \pm 17$ & $0.842 \pm 0.019$ & 0.94 \\
& 3.0 & $(4.82 \pm 0.29) \times 10^{-3}$ & $372 \pm 16$ & $0.947 \pm 0.014$ & 1.99 \\
\hline
\end{tabular}




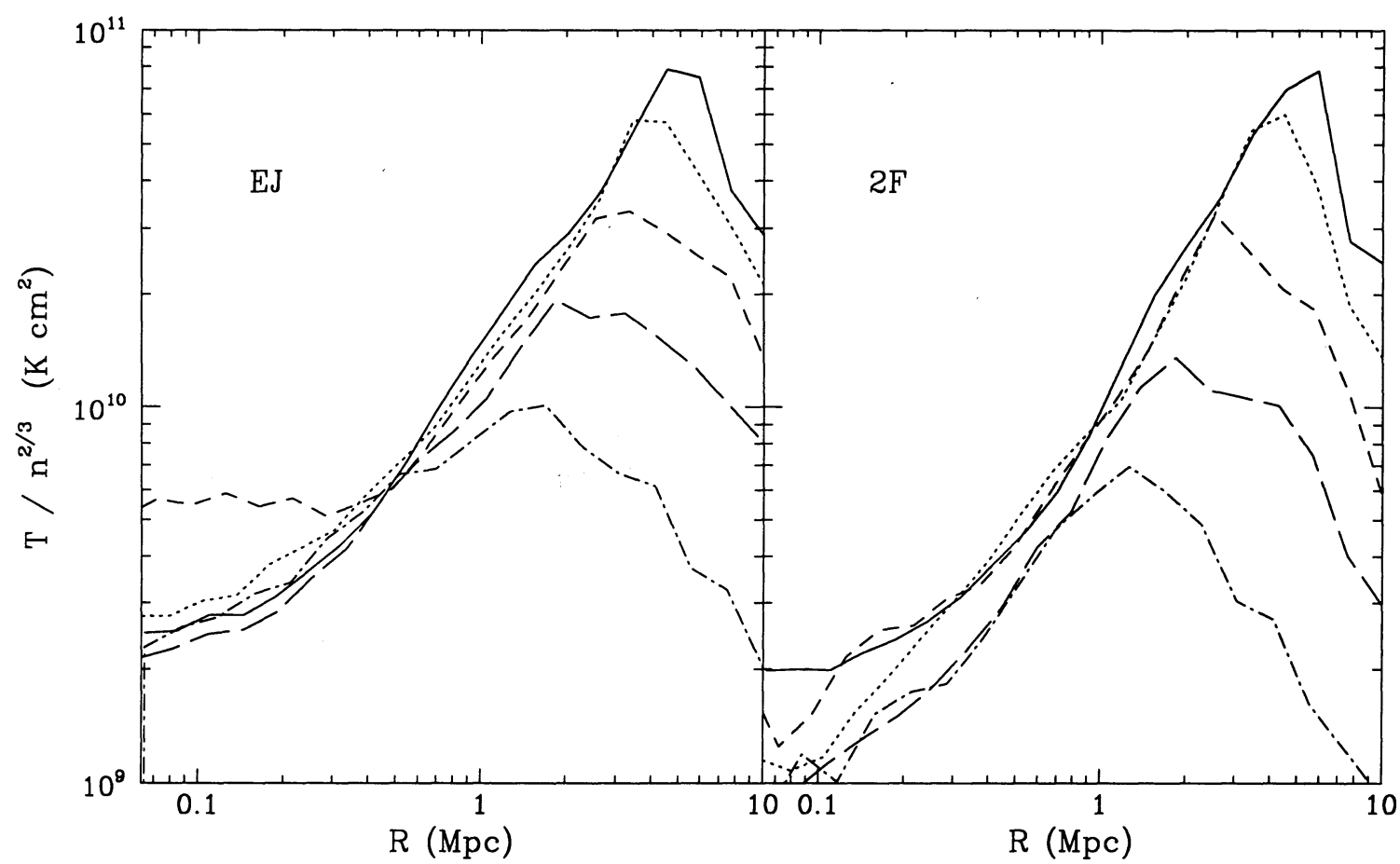

Fig. 4.-Entropy profile of the cluster gas for the two runs, evaluated at the same redshifts as Fig. 2 with the same linestyles. The appearance of a jump in central entropy for the $z=0.246$ profile is a consequence of centering on the most bound dark matter particle; at the epoch, the potential minimum is briefly offset from the low entropy gas because of merger activity, as described in the text.

larger than for the dark matter and gas distributions. The steeper galaxy density profile is in agreement with the fact that the galaxy population has a lower velocity dispersion (i.e., is cooler) than the dark matter in the cluster, as discussed in the following section. It is also in agreement with observations. The ratio of mass in gas to mass in cluster galaxies, in the ejection run, is less than one within $300 \mathrm{kpc}$, and rises steeply

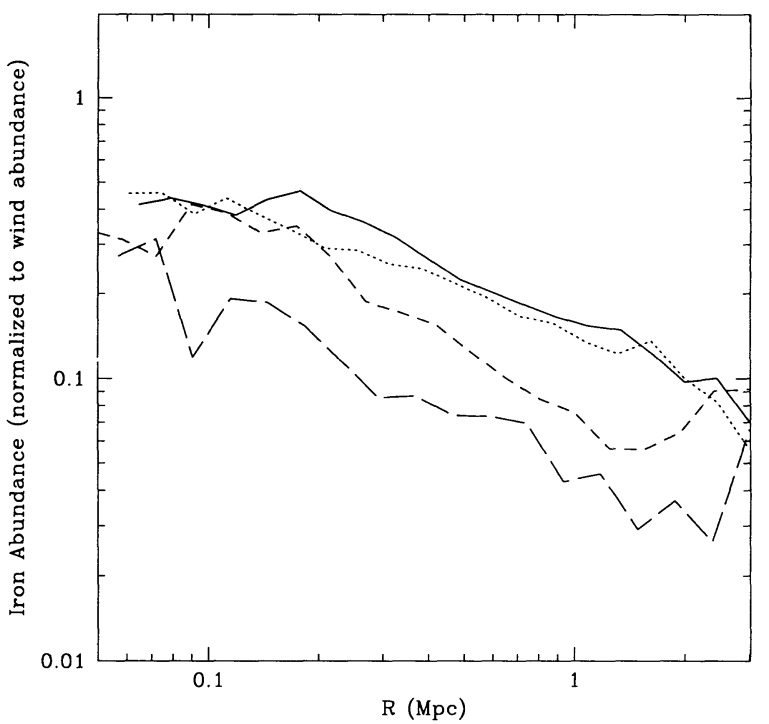

FIG. $5 a$ beyond to a value of 5.9 at an Abell radius. This is comparable to the value of 5.1 inferred for the Coma cluster within that radius (White et al. 1993).

The radial distribution of ejected gas in the cluster can be used to define an iron abundance profile. Figure $5 a$ shows the true iron abundance profile of the intracluster medium, normalized to the galactic wind abundance, at four redshifts. The

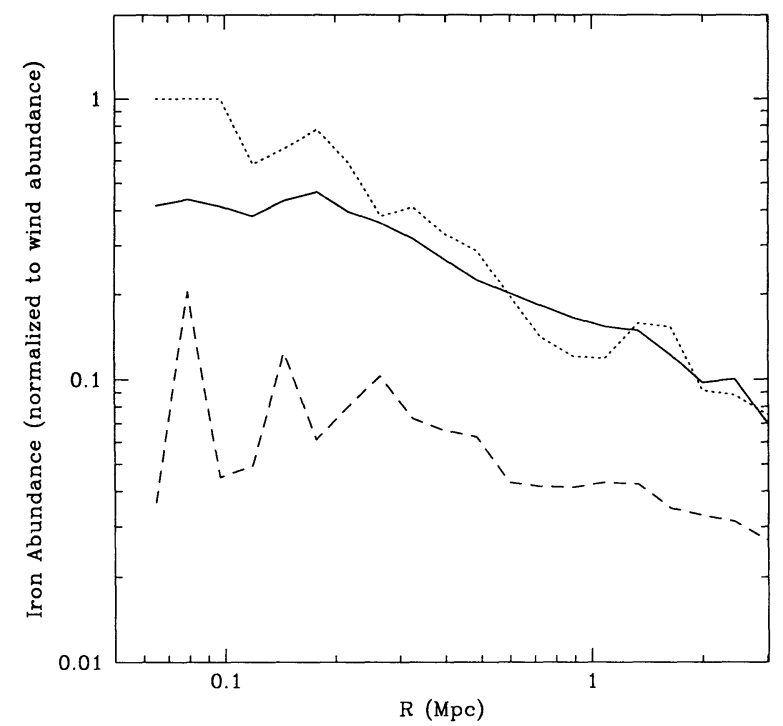

FIG. $5 b$

FIG. 5.-Iron abundance profiles for the cluster. (a) The iron abundance profile of the cluster, at four different redshifts: $z=0.02$ (solid curve), $z=0.102$ (dotted curve), $z=0.499$ (short-dashed curve), and $z=1.01$ (long-dashed curve). (b) The iron abundance profile of the cluster at a redshift of $z=0.02$, for different galaxy ejection histories. The solid line refers to the standard model shown in (a) Also shown are a model with the same history, but assuming no mixing of metals with surrounding gas at the time of ejection (dot-dashed curve) and a model with $\sim 25 \%$ as much mass and energy ejected, at a flat rate between redshifts of four and one (dashed curve). 
profiles for $z=0.499$ and $z=1.01$ were obtained from the most massive progenitor of the final cluster. The jagged nature of the profiles in the center is due to discreteness noise. A clear metallicity gradient is present, even at a redshift of one.

The normalization of the metal abundance is tied to the wind abundance. If the latter is taken to be three times the solar value, the average abundance for all gas within $318 \mathrm{kpc}$ (about a core radius) is 1.62 solar; one must go beyond $2 \mathrm{Mpc}$ for the average abundance within to drop below half solar. Meanwhile, typical ICM iron abundances are on the order of one-third solar. The simulation abundances could be decreased in three ways: by decreasing the metallicity of the ejecta; by decreasing the amount of ejected matter; and by adopting a time-varying ejection rate and ejecta iron mass fraction. Most probably, some combination of the latter two is in order.

Recall that changing the metallicity of the ejecta in our simulation amounts only to rescaling the vertical axis of Figure $5 a$ by a constant factor. Similarly, changing the total amount of mass ejected-but keeping a flat ejection rate-will only change the fraction of the ICM made up of ejected matter by a constant factor. In other words, so long as the ejection rate and metallicity of ejecta are kept uniform in time, the shape of the true iron abundance profile should be the same in this simulated cluster; at $z=0.02$, the iron abundance at one core radius should be $\sim 3$ times its value at three core radii.

As for different galaxy ejection histories, Figure $5 b$ shows the iron abundance profile at a redshift of 0.02 for three different runs - our standard run, the run with no mixing, and the run with truncated ejection. The strength of the abundance gradient is fairly insensitive to the assumption of mixing. The early ejection run displays the same gradient when expressed in terms of the ratio of the abundance at, say, $1 \mathrm{Mpc}$ compared to $300 \mathrm{kpc}$. However, since the overall abundance is down by a factor of $\sim 4$, observational detection of this gradient would be more difficult. Note that all models show a negative radial gradient. This suggests that observational evidence for central concentration of iron is not enough to convincingly argue that ram pressure stripping of cluster galaxies was the mechanism for its deposition; it is possible to obtain strong gradients through energetic ejection.

Why does the gradient exist? In Figure 3, the galaxy number density profile at the beginning of the simulation shows a shallow gradient from the comoving location of the center of the final cluster; this shallow gradient steepens by $z=0.02$, when the fall-off in galaxy number density is faster than the fall-off of intracluster gas density. Because the galaxies are the source of ejected matter, it seems reasonable to expect that the more steep galaxy density profile (relative to the intracluster gas) would lead to a metallicity gradient. There are certainly other processes which could play a role. For instance, since the gas is ejected at roughly constant temperature in this model, one could envision ejected gas which was cooler or hotter than its surroundings moving inward or outward to a region of comparable entropy. However, the difference in density profiles seems a strong enough effect to easily account for the gradient. Figure 6 shows the profiles of three ratios: (1) the mass in galaxies within a radius $r$ over the primordial gas mass within $r$, at $z=9.0$; (2) the same quantity at $z=0.02$; and (3) the mass in ejected gas within $r$ over the primordial gas mass within $r$, at $z=0.02$. These ratios are profiled against the total mass enclosed within $r$. In the absence of mixing between shells of constant mass enclosed and any strong radial motion of the

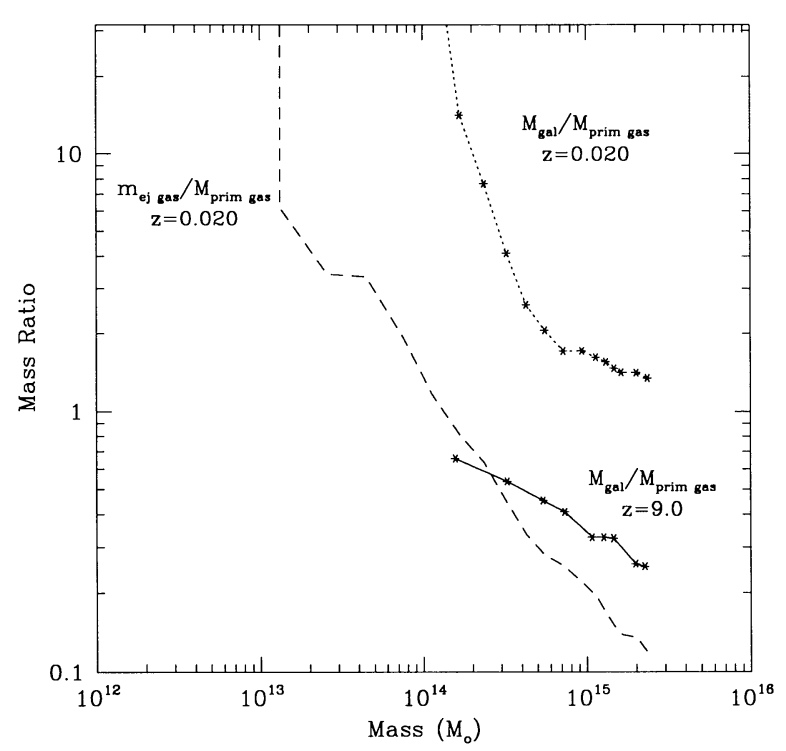

Fig. 6.-Value of three mass ratios plotted against enclosed mass (against radius which encloses that mass): mass in galaxies over mass in (necessarily primordial) gas at $z=9.0$ (solid curve); mass in galaxies over mass in primordial gas at $z=0.02$ (dotted curve); mass in ejected gas over mass in primordial gas at $z=0.02$ (dashed curve).

ejected matter, one would expect the shape of the profile for ratio 3 to be similar to that of ratio 2 . The metallicity profile in Figure 5 appears to begin levelling off at $\sim 1 \mathrm{Mpc}$ for $z=0.02$; that corresponds to an enclosed total mass of $\sim 4.66 \times 10^{14}$ $M_{\odot}$. Within that mass shell, ratios 2 and 3 appear to have similar shapes.

To summarize, the metal gradient appears to be a consequence of ejection which takes place while galaxies have a gradient in number density with respect to primordial gas. The latter is due to the fact that the ICM is slightly hotter and therefore more extended than the galaxy distribution.

The two rich clusters with data on abundance gradients are Coma and Perseus. Observations have suggested that Coma has a fairly flat iron abundance profile (Hughes, Gorenstein, \& Fabricant 1988; Hughes et al. 1992; Watt et al. 1992). Imaging of the Perseus cluster by Spacelab 2 (Ponman et al. 1990) and Spartan 1 (Kowalski et al. 1993) suggest a strong abundance gradient, decreasing with radius; however, analyses of BBXRT data disagree with this result (Mushotzky 1991). It has been suggested (e.g., Watt et al. 1992) that a lack of an abundance gradient in Coma, compared with a strong gradient in Perseus, is indicative of a difference in formation history; clusters that formed recently via major mergers would have any preexisting abundance gradients "washed out" in the merger. Such mixing after mergers was examined for collisionless fluids such as stellar systems by White (1980), who found mixing to be relatively inefficient. We examine this hypothesis for the collisional intracluster gas by looking at the iron abundance profiles of the objects that merge to form the final cluster, and the merger product, over a time period around the merger. The abundance profiles for the three subclusters that merge between $z=0.499$ and $z=0.246$ (see Fig. 1), along with their evolution over a period of $1.47 \mathrm{Gyr}$, are shown in Figure 7. Lines depicting an object disappear when they merge into the main cluster. A strong gradient in the most massive object is clearly in place by a redshift of 0.401 ; a subsequent merger with the next most massive subcluster does not seem to diminish that gradient. 


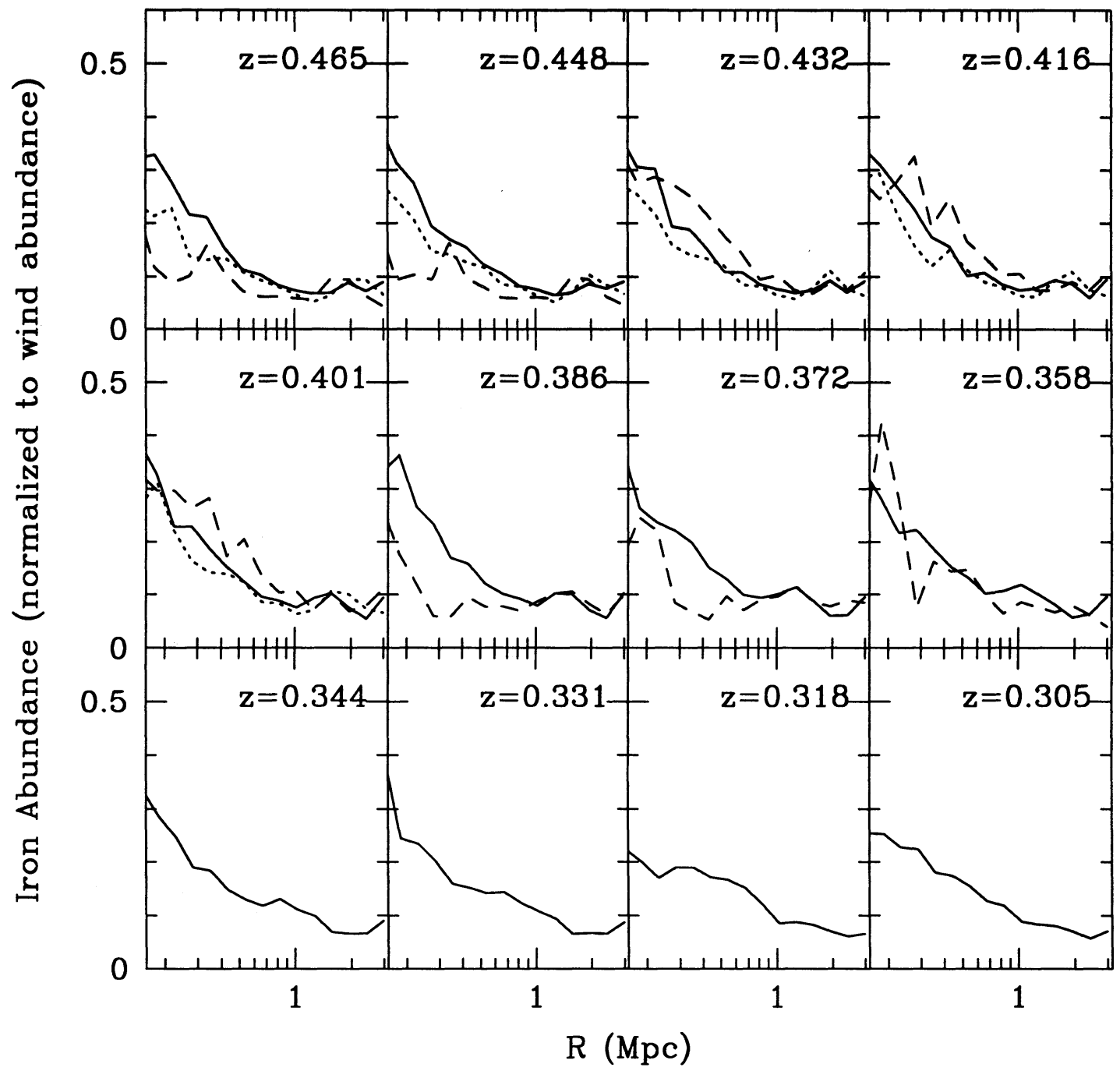

Fig. 7.-Evolutionary sequence of iron abundance profiles for three protocluster objects which merge over the time period shown. The solid line corresponds to the largest progenitor of the three at $z=0.465$, the dashed line to the smallest. Lines disappear when they merge into the more massive object. Merging does not strongly affect the abundance gradient.

The second merger also seems to have little effect. We therefore can claim to see no clear evidence for such a relationship between abundance profile and recent dynamical history in our simulation.

However, it should be noted that the profiles displayed in Figures 5 and 7 are true mass-weighted radial profiles, based on the average abundance in concentric spherical shells centered on the minimum in the potential. Observers do not have access to this information; observed profiles are necessarily projected and flux-weighted. Furthermore, they are routinely centered on or taken from the $\mathrm{X}$-ray surface brightness maximum rather than the potential minimum, which is unknown. While the cluster gas attempts to relax after mergers, the surface brightness maximum, the iron abundance maximum, and the potential minimum are often in three different projected locations. This is the source of the "weaker gradient" at $z=0.318$; the profile is centered on the potential minimum, which is far from the abundance maximum, so the abundance maximum gets averaged over a large spherical shell and does not show up in the profile. As the surface brightness and iron abundance maxima are near each other at that red- shift, a flux-weighted profile centered on the surface brightness maximum continues to show the strong gradient. While the offsets between the two maxima, and the potential minimum, complicate the definition of a "central" iron abundance, this effect does not appear here to be able to make Coma appear to have a flat abundance profile. The two maxima are always near enough to each other that a value of the flux-weighted iron abundance at the surface brightness peak is still large, even if not the largest; values at $20^{\prime}$ away are generally depressed relative to that central value.

Alternatively, the apparatus used to generate X-ray images and surface brightness profiles (described below) can also be used to create a projected, flux-weighted metallicity map. This map indicates the iron abundance an observer would measure if the observer could obtain a spectrum from every location in the map. Sampling the map at various points then creates abundance profiles more akin to those from real observations.

Figure 8 shows the results of this process for the cluster at $z=0.020$. The upper left-hand corner is a flux-weighted metallicity map, using emission in the ROSAT passband, with angular resolution of 0.5. The central concentration of metals 
is evident in this image, as is the fact that the contours are fairly well aligned with the cluster galaxies. Below this is such a map for the same cluster, with no mixing of ejected metals taking place at the time of ejection. There is significantly more struc! ture in the metal distribution when mixing is suppressed. Note that flux-weighting along the line of sight has diminished the observed central iron abundance to $\sim 70 \%$ of the wind abundance.

The right-hand panels of Figure 8 show flux-weighted metallicity maps using emission in the passband of $2-10 \mathrm{keV}$, the same as EXOSAT and $H E A O$ 1. The pixels in this map were generated by flux-weighting along the line of sight assuming an instrument response modeled by a Gaussian filter with a FWHM of 45'. Note the different contour levels used from the ROSAT map. After smoothing, the central iron abundance has dropped to almost $35 \%$ of the wind abundance, and the structure in the projected metallicity evident above has vanished. Also, the apparent abundance maximum is now over $10^{\prime}$ from the ROSAT X-ray surface brightness maximum. The "nomixing" EXOSAT map makes clear that with this heavy smoothing, the iron abundance profile can appear quite flat, when in reality a strong gradient exists. The strong-mixing model, observed with EXOSAT, shows no evidence for an abundance gradient although one is present.

\section{4. $X$-Ray Characteristics}

To study the X-ray emission that would be expected from our simulated clusters, we integrated a band-limited thermal bremsstrahlung emissivity over the volume of interest by using the equations of SPH to turn the volume integral into a sum over particles (see Appendix).

Figure 9 shows the redshift evolution of the (appropriately redshifted) band-limited X-ray luminosity for the ejection and two-fluid runs. To emphasize evolutionary effects, we show the ratio $L_{x}(z) / L_{x}(0)$. The final $\mathrm{X}$-ray luminosities are $L_{x}(0)=2.4$ and $1.3=10^{44} \mathrm{ergs} \mathrm{s}^{-1}$ for the $2 \mathrm{~F}$ and EJ runs, respectively. At hgih redshift, the luminosity from the two-fluid run is as much as a factor of five greater than for the ejection run. It remains roughly constant until $z \approx 0.5$. The slow accumulation of matter during this time period does not significantly affect the $\mathrm{X}$-ray luminosity. After $z \approx 0.5$, the luminosity climbs sharply, the result of a large merger in-progress. Both of the peaks/

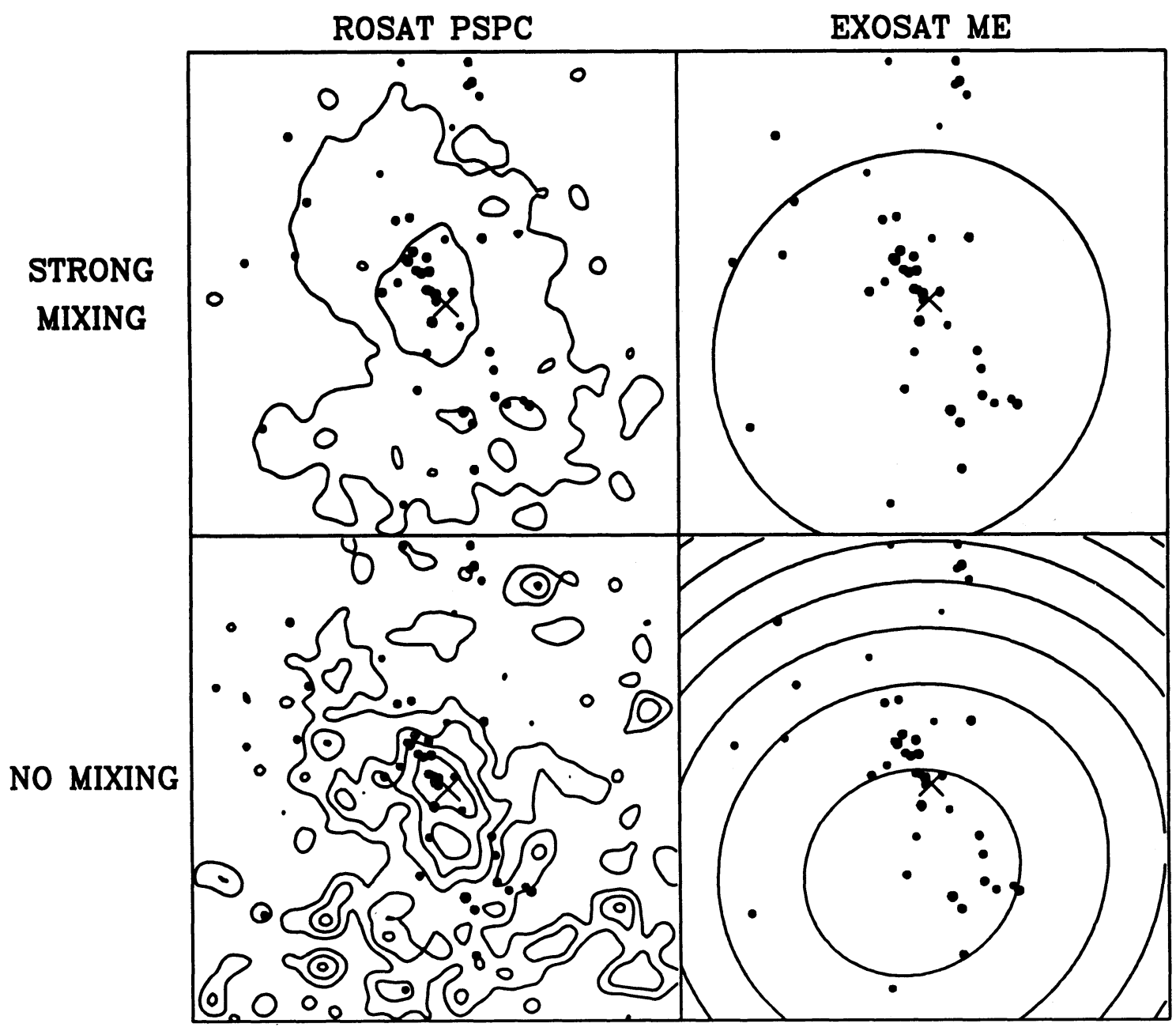

Fig. 8.-Four projected, flux-weighted iron abundance maps of the simulated cluster. Two ejection histories are shown: the flat model used throughout the paper and a second model with the same history, but assuming no mixing of metals with surrounding gas at the time of ejection. The maps are constructed using the passband and resolution of the ROSAT PSPC and EXOSAT ME detectors. The abundances are normalized to the wind abundance, with contour levels of $0.14,0.28$, $0.42,0.56$, and 0.70 (ROSAT PSPC) and 0.25, 0.27, 0.28, 0.30, 0.32, 0.33, and 0.35 (EXOSAT ME). 


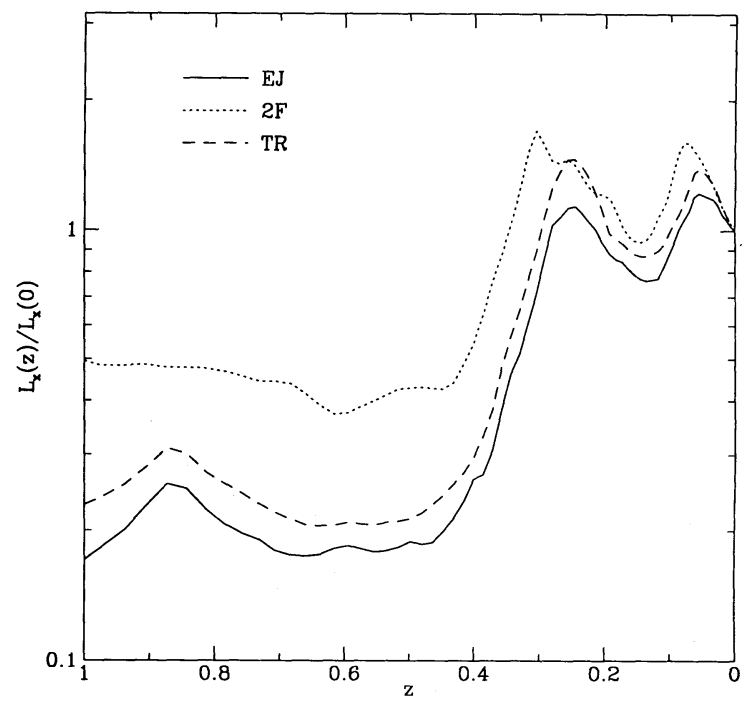

Fig. 9.-Evolution of $L_{x}$, within a comoving $1 \mathrm{Mpc}$, band-limited to the ROSAT PSPC passband, with $z$, for ejection run (solid line) and two-fluid run (dotted line).

valleys at lower redshifts correspond to merger events. Again, similar to Evrard (1990b), we see that the X-ray luminosity can decrease after mergers due to oscillations in the central density as the cluster attempts to relax.

From $z=1$ to the present, the EJ run luminosity increases by a factor of $\sim 6.5$ whereas the $2 \mathrm{~F}$ run grows by a factor of 2.3. The larger increase in the EJ run is a consequence of the fact that the "preheating" by gas ejection affects primarily the central, low adiabat gas. At early times, preheating drives gas onto higher adiabats, and the emission from the core of the cluster is necessarily diminished. As gas piles onto the cluster at larger radii and higher adiabats, the relative importance of the core emission diminishes, and the difference between the EJ and $2 \mathrm{~F}$ runs decreases. An additional increase in the EJ run arises from the fact that mass is continually being added to the cluster from galaxy winds. The mass of gas added by galactic winds between $z=1$ and the present amounts to $\sim 11 \%$ of the final ICM mass.

The luminosity evolution of the run with truncated winds lies intermediate between the EJ and $2 \mathrm{~F}$ models, with a factor of $\sim 4$ increase since $z=1$. This run has no mass added to the ICM over this period, so the effects on the luminosity evolution are solely due to preheating.

Energetic winds thus imply lower luminosities at high redshift $(z \sim 1)$, combined with more rapid evolution to $z \sim 0$, than otherwise would take place in hierarchical models. Rapid evolution in X-ray luminosity has been hinted at in the observations of Castander et al. (1993). Unfortunately, the small number of clusters in their sample (5) makes it difficult to conclusively rule out models. However, the fact that they detected emission from 3 of the clusters at $z \sim 1$ should provide an upper limit to the strength of feedback from galactic winds, since extremely strong input would make the clusters invisible at high redshift.

Figure $10 a$ shows a sequence of simulated ROSAT PSPC images for the run with galaxies and ejection, while Figure $10 b$ shows corresponding images for the $2 \mathrm{~F}$ run. The evolution of the model proceeds clockwise from the upper left. The outer surface brightness contour, and the spacing between contours, are the same for both figures and throughout each figure. The black dots in the run with galaxies/ejection corresponds to the projected locations of the galaxies in the simulation. The calculated incident flux in the $0.1-2.4 \mathrm{keV}$ band was converted to ROSAT PSPC counts per second using a simple conversion factor taken to be (ROSAT Scientific Data Center, 1989)

$$
\text { 1.4 PSPC counts s} \mathrm{s}^{-1}=1 \times 10^{-11} \mathrm{ergs} \mathrm{s}^{-1} \mathrm{~cm}^{-2} \text {, }
$$

for a thermal spectrum in this temperature range. The "exposure" was simulated as $7.2 \mathrm{ks}$ in length. Because of the preheating, there are sources of X-ray emission in the $2 \mathrm{~F}$ run visible at high redshift that are too dim to be seen in the run with ejection. At lower redshifts, the size of the main object is roughly comparable in the two runs, but there are more contours in the $2 \mathrm{~F}$ model. The ejection run achieves higher cluster temperatures, but lower densities, and thermal bremsstrahlung emission is more strongly sensitive to the density of the gas.

The stronger emission shown in Figure $10 b$ allows us to see the mergers implied in the luminosity evolution (Fig. 9). Of the four central sources of emission at $z \approx 0.5$, the three northern sources merge between $z=0.5$ and $z=0.3$; this is the source of the first peak in Figure 9, and the strong central source at $z \approx 0.25$ in Figure $10 b$. The source to the south merges at $z \approx 0.1$, but still retains some dynamical identity. Thus, in addition to the second peak on Figure 9, we see the $z=0.102$ isophotal contours in Figure $10 b$ as stretched to the southeast. Momentum from this sublump subsequently drives north, causing the contour lines to be stretched in that direction at later redshifts, as has been seen in other simulations examining mergers (e.g., Roettiger, Burns, \& Loken 1993).

The images shown have been fitted to a radially symmetric surface brightness profile of the usual form implied by the $\beta$-model,

$$
\Sigma_{x}(\theta)=\Sigma_{0}\left[1+\left(\theta / \theta_{x}\right)^{2}\right]^{-3 \beta_{\mathrm{fit}}+1 / 2} .
$$

Poisson noise was added at an amplitude of $3 \times 10^{-4}$ counts $\mathrm{s}^{-1} \operatorname{arcmin}^{-2}$; a flat background of this value was then subtracted off. Only bins with surface brightnesses higher than this value were used in the fits. Figure 11 shows the values and errors for the surface brightness in each radial bin, as well as the best fit, for the four lowest redshifts imaged in each run. Table 4 shows the best fit values and errors for the central surface brightness $\Sigma_{0}$, X-ray core radius $r_{x}$ (for $\theta_{x}$ at redshift $z$ ), and exponent $\beta_{\mathrm{fit}}$, for the eight fits. In all cases, equation (10) provides a good fit to the surface brightness profiles. At redshifts $z \lesssim 0.1$, the values of the fitting parameters are comparable to those obtained in rich cluster observations.

TABLE 4

X-Ray Surface Brightness Profile Fits

\begin{tabular}{ccclc}
\hline \hline Run & Redshift $z$ & $\begin{array}{c}\Sigma_{0} \\
\left.\text { (counts s }{ }^{-1} \text { arcmin }^{-2}\right)\end{array}$ & $\begin{array}{c}r_{x} \\
(\mathrm{kpc})\end{array}$ & $\beta_{\text {fit }}$ \\
\hline EJ $\ldots \ldots$ & 0.25 & $(1.07 \pm 0.09) \times 10^{-2}$ & $839 \pm 301$ & $1.882 \pm 1.046$ \\
& 0.10 & $(1.80 \pm 0.10) \times 10^{-2}$ & $294 \pm 24$ & $0.681 \pm 0.042$ \\
& 0.06 & $(3.01 \pm 0.07) \times 10^{-2}$ & $409 \pm 15$ & $0.929 \pm 0.032$ \\
& 0.02 & $(3.88 \pm 0.04) \times 10^{-2}$ & $282 \pm 4$ & $0.741 \pm 0.008$ \\
& & & & \\
& & & & \\
& 0.25 & $(2.54 \pm 0.14) \times 10^{-2}$ & $778 \pm 115$ & $1.776 \pm 0.376$ \\
& 0.10 & $(5.68 \pm 0.17) \times 10^{-2}$ & $295 \pm 12$ & $0.769 \pm 0.024$ \\
& 0.06 & $(8.99 \pm 0.15) \times 10^{-2}$ & $267 \pm 5$ & $0.734 \pm 0.010$ \\
& 0.02 & $(8.99 \pm 0.07) \times 10^{-2}$ & $271 \pm 2$ & $0.788 \pm 0.005$ \\
\hline
\end{tabular}




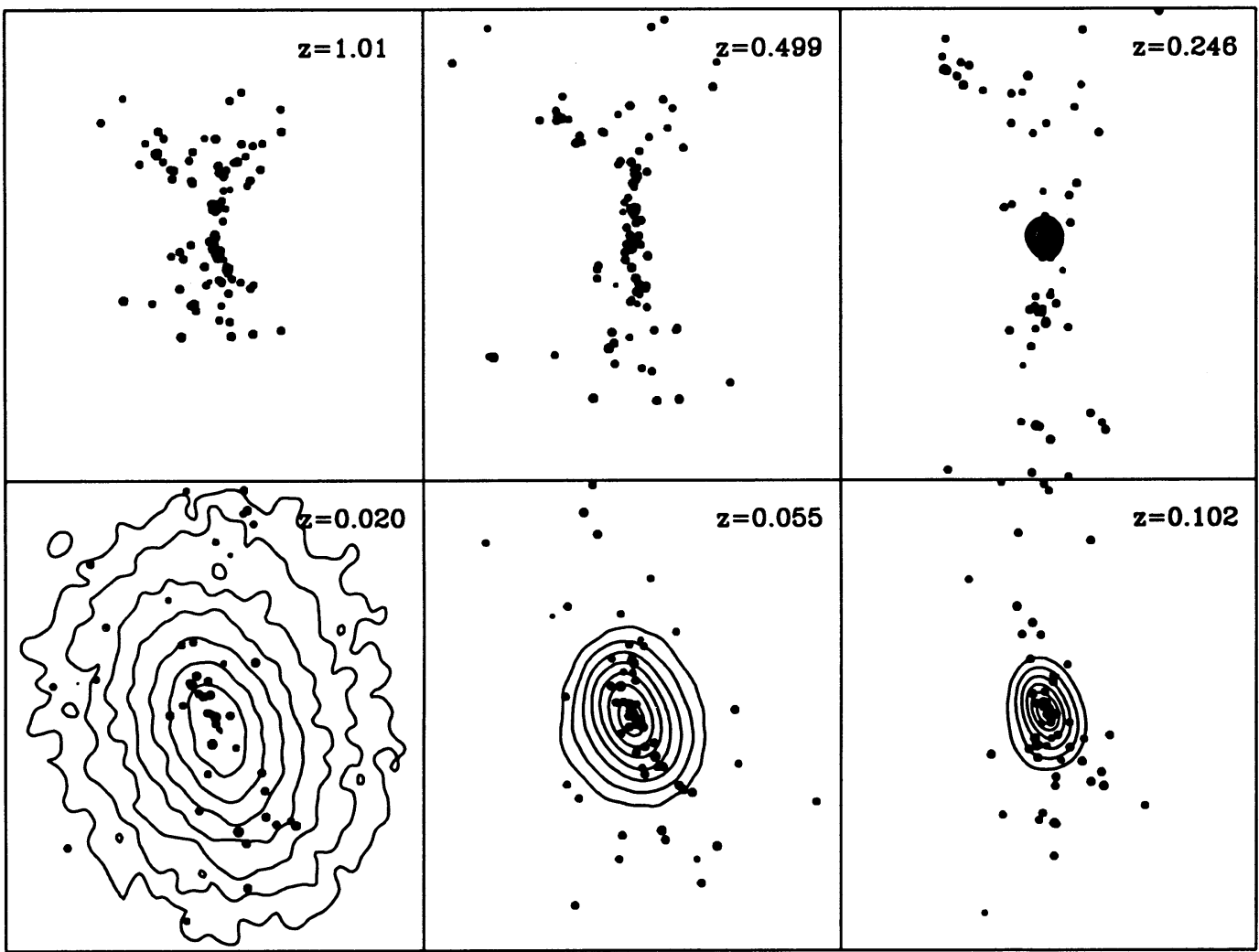

FIG. $10 a$

Fig. 10.-Evolutionary sequence of simulated ROSAT PSPC X-ray images for both runs, in $z$-axis projection at the indicated redshifts. $(a)$ is ejection run; $(b)$ is

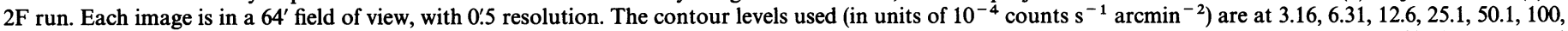

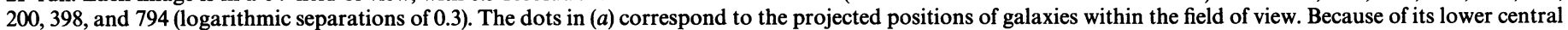

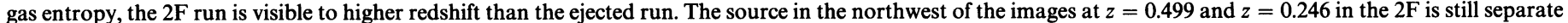
(has not merged) at the redshifts of the later images; it is outside the field of view at those redshifts.

The values of $\beta$ at $z=0.02$ derived from imaging are consistent with, but not exactly equal to, the value for $\alpha_{\text {ICM }}$ obtained above from directly fitting the three-dimensional density profile. The errors are smaller for the surface brightness measurement because they are based on Poisson statistics of photon counts. The difference is not surprising given the level of asymmetry in the X-ray profiles. The $\beta_{\text {fit }}$ value of $0.74 \pm 0.01$ for the EJ run is again smaller than the $0.79 \pm 0.01$ value for the $2 \mathrm{~F}$ run. However, this ranking does not hold at all times. The previous output frame at $z=0.06$ shows the EJ profile is steeper than the $2 F$ profile. Evidently, the effect on $\beta_{\mathrm{fit}}$ due to feedback is not larger than the variance caused by dynamical effects.

\section{MASS ESTIMATES, THE CLUSTER BARYON FRACTION, AND THE $\beta$-PROBLEM}

\subsection{X-Ray and Optical Mass Estimates}

Under the $\beta$-model, it is assumed that the gas density is well described by equation (7), with $\beta$ and $r_{c}$ obtained from the cluster's X-ray surface brightness profile. Furthermore, by assuming the gas is isothermal, the central surface brightness can be simply related to the emission measure along a line of sight through the center of the cluster, and thus to the central gas density. This leads to a relation between central number density $n_{0}$ and X-ray observables of the form

$$
\begin{aligned}
n_{0}= & 1.34 \times 10^{-3} \mathrm{~cm}^{-3} \frac{(1+z)^{2}}{\left[f_{\text {band }}(T, z)\right]^{1 / 2}} \frac{\Gamma(3 \beta)}{\Gamma(3 \beta-1 / 2)} \\
& \times\left(\frac{\Sigma_{0}}{10^{-2} \text { counts s }^{-1} \operatorname{arcmin}^{-2}}\right)^{1 / 2} \\
& \times\left(\frac{T}{10^{8} K}\right)^{-1 / 2}\left(\frac{r_{c}}{250 \mathrm{kpc}}\right)^{-1 / 2},
\end{aligned}
$$

where we have figured in our ROSAT response approximation, equation (9); and $f_{\text {band }}(T, z)$ is the fraction of emission that falls into the ROSAT passband (see Appendix). Thus, $\Sigma_{0}, \beta, r_{c}$, and the cluster temperature give the central density $n_{0}$; integrating the density above gives the gas mass within a certain radius.

Assuming that the ICM is both hydrostatic and ideal, the total mass within a given radius is given by

$$
M(<r)=\frac{k T(r) r}{\mu m_{p} G}\left(-\frac{d \ln \rho_{g}}{d \ln r}-\frac{d \ln T}{d \ln r}\right) .
$$

If the gas is isothermal and the density follows equation (7), we have

$$
M_{\text {bind }}(<r)=\frac{k T(r) r}{\mu m_{p} G} 3 \beta \frac{\left(r / r_{c}\right)^{2}}{1+\left(r / r_{c}\right)^{2}}
$$




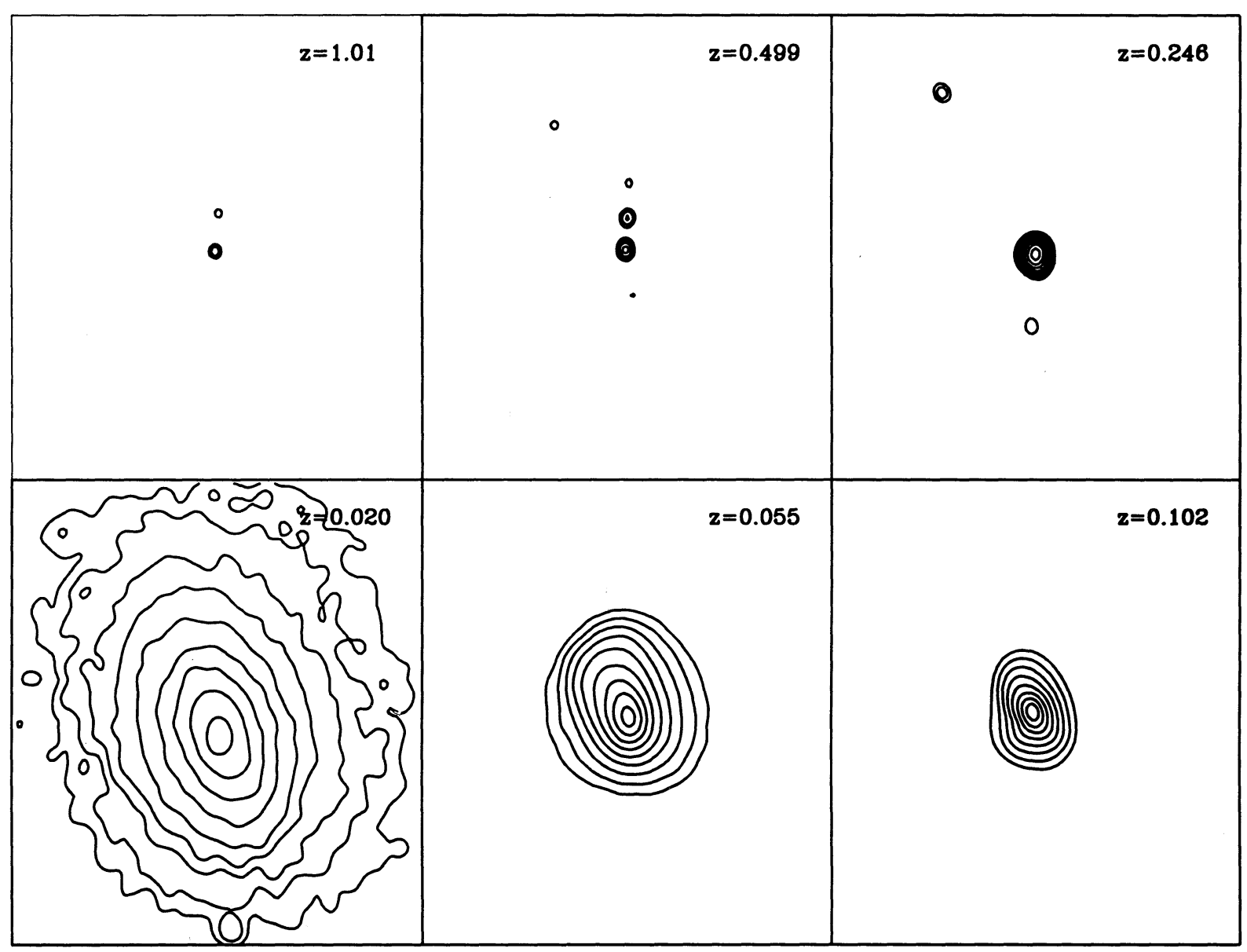

FIG. $10 b$

For large radii $\left(r \gg r_{c}\right)$, the last fractional factor goes to 1 and can be ignored; but for smaller radii, ignoring this factor will result in overestimates of the binding mass. At $r=3 r_{c}$, for instance, a $10 \%$ error is introduced.

To compare the results of the simulation with such a binding mass estimate, an additional correction to this equation is necessary. Equation (12) was derived assuming the usual hydrostatic equation, which in turn assumes an $r^{-2}$ force law. However, the force law used in the simulations comes from the softened point-mass potential, $V(r) \propto\left(r^{2}+\epsilon^{2}\right)^{-1 / 2}$, and not a Coulomb law. Furthermore, for such a force, Gauss's law no longer holds; the importance of the correction depends on the distance away from the cluster center. Thus, the hydrostatic equation becomes

$$
\frac{\nabla P}{\rho}=-S(r / \epsilon) \frac{G M r}{\left(r^{2}+\epsilon^{2}\right)^{3 / 2}} \hat{r},
$$

where $S$ is a function that depends on an integral over the mass distribution. Our densities at large radii are roughly proportional to $r^{-2}$, and with such a distribution, $S$ is approximately unity. The formula for estimating binding masses in our simulations is then

$$
M(<r)=\frac{k T(r) r}{\mu m_{p} G} 3 \beta \frac{\left(r / r_{c}\right)^{2}}{1+\left(r / r_{c}\right)^{2}}\left[1+(\epsilon / r)^{2}\right]^{3 / 2} .
$$

Figure 12 shows the actual gas mass (boxes) and total mass (crosses) contained within, for the ejection run at a redshift of $z=0.02$. Also shown for comparison are predictions for the enclosed gas and total masses, described above. The values of $\beta, r_{c}$ (or $r_{x}$ ), and $\Sigma_{0}$ (thus $n_{0}$ ) are from the corresponding $z$-axis projection surface brightness fit (see Fig. 11 and Table 4). Similar results are found for the other two projections. The temperature used in the binding mass estimate is the "central temperature" at that redshift from the true profile discussed in $\S 3.3$ above. Both the inferred gas and binding masses are accurate to better than $20 \%$ beyond $500 \mathrm{kpc}$. There is a tendency to underestimate the binding mass which grows larger at smaller radii. However, at scales above our resolution limit, the error in this mass estimate is never larger than $\sim 45 \%$, comparable to the error found in two-fluid simulations (Evrard, Metzler, \& Navarro 1994). The introduction of the additional physics of galactic winds does not detrimentally affect the accuracy of mass estimates drawn from the $\beta$-model.

Optically, one can obtain a binding mass estimate using the first velocity moment of the collisionless Boltzmann equation. The "hydrostatic equation" for galaxies can be written as

$$
M(<r)=-\frac{r^{2}}{G \rho_{\mathrm{gal}}} \frac{d\left(\rho_{\mathrm{gal}} \sigma_{r}^{2}\right)}{d r}-\frac{2 r \sigma_{r}^{2} A(r)}{G},
$$

where $\sigma_{r}$ is the radial velocity dispersion, and $A(r)=1-\sigma_{t}^{2} / \sigma_{r}^{2}$ is the anisotropy parameter. If we further assume that cluster velocity dispersions are isotropic $[A(r)=0]$ and independent of 


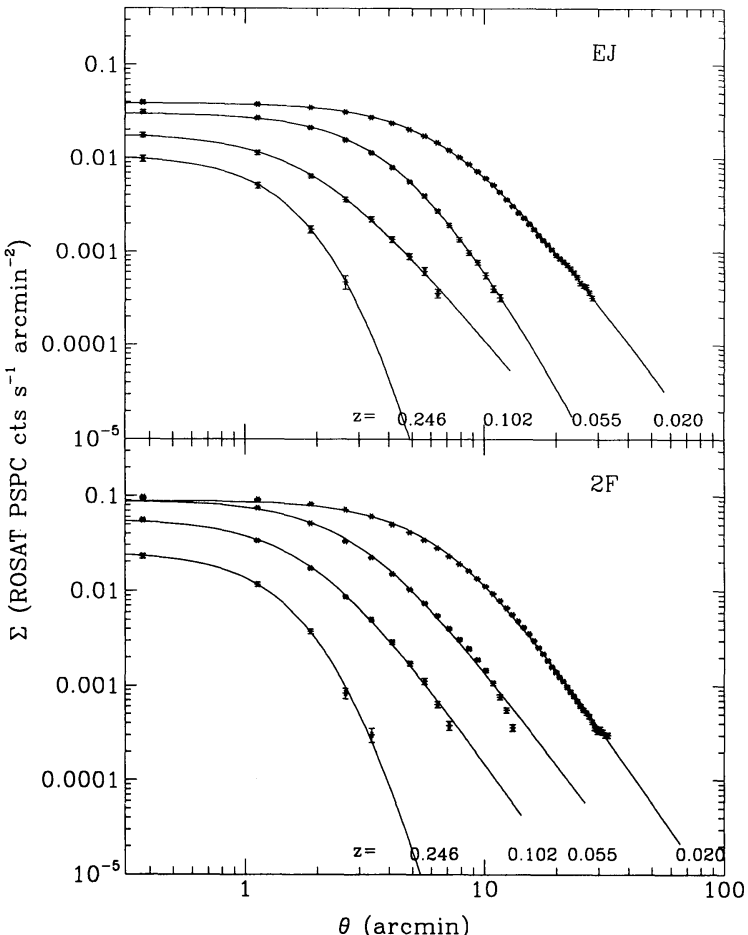

Fig. 11.- Surface brightness profiles for both runs, at the four lowest redshifts imaged in Fig. 10. Also shown are fits to the $\beta$-model. No data are shown where the surface brightness drops below the fiducial choice for the background of $3 \times 10^{-4}$ counts s $\mathrm{s}^{-1}$ arcmin $^{-2}$. Table 4 gives the best-fit parameter values and errors.

radius, we get

$$
M(<r)=\frac{r \sigma_{r}^{2}}{G}\left(-\frac{d \ln \rho_{\mathrm{gal}}}{d \ln r}\right) .
$$

Assuming $\rho_{\mathrm{gal}}$ is well fitted by equation 7 , and fitting the galaxy density profile out to $3 \mathrm{Mpc}$, the logarithmic derivative $-d \ln \rho_{\mathrm{gal}} / d \ln r \sim 3 \alpha_{\mathrm{gal}}=2.7$. If we plug in the line-of-sight velocity dispersions of 606,960 , and $655 \mathrm{~km} \mathrm{~s}^{-1}$ obtained by viewing the cluster along the simulation's principal axes (drawn from 61, 94, and 64 galaxies within the projected Abell radius), we get mass estimates (in units of $10^{15} M_{\odot}$ ) of $0.69,1.7$, and 0.81 , respectively. The actual mass within an Abell radius is $1.2 \times 10^{15} M_{\odot}$. The inaccuracy is due to error in our assumptions of isotropy or radially constant velocity dispersions. Additionally, for one projection, the infalling subcluster apparent in Figure 1 falls within the projected $3 \mathrm{Mpc}$ radius; a large number of infalling objects boosts the sample size but artificially inflates the estimate of velocity dispersion. Interestingly, if we abandon the isotropy assumption and avoid projection effects by using an average one-dimensional velocity dispersion determined from real three-dimensional data of 769 $\mathrm{km} \mathrm{s}^{-1}$, we obtain an estimate of $M(<3 \mathrm{Mpc})=1.2 \times 10^{15}$ $M_{\odot}$ equal to the true value. Again, however, this agreement is fortuitous and, at any rate, impossible to measure observationally.

Another method of estimating virial masses is by use of a projected estimator, such as equation (10-23) in Binney \& Tremaine (1987):

$$
M_{\mathrm{vir}}=\frac{3 \pi N^{2} \sigma^{2}}{2 G} \frac{1}{\sum_{i=1}^{N} \Sigma_{j<i}\left|R_{i}-R_{j}\right|^{-1}},
$$

with $N$ the number of galaxies, $\left|\boldsymbol{R}_{i}-\boldsymbol{R}_{j}\right|$ the projected separation between galaxies $i$ and $j$, and $\sigma$ the one-dimensional velocity dispersion as usual. Applying this estimator to our cluster along the same three axes yields mass estimates (in units of $10^{15} M_{\odot}$ ) of $0.49,1.3$, and 0.58 , respectively. This technique is known to underestimate binding masses where the dark halo is more extended than the galaxy distribution, as is the case here. For the second axis, this underestimate is countered by the overestimate of $\sigma$ due to projection effects from the infalling subcluster, resulting in a mass estimate which is actually close to the mark, but is nonetheless spurious.

\subsection{The Cluster Baryon Fraction}

The observed baryon fraction of Coma and other rich clusters appears to exceed the limits on the global baryon fraction set by nucleosynthesis constraints if $\Omega=1$ (White et al. 1993). Above, we described how one obtains estimates of the gas and binding mass within a certain radius from X-ray observations. Galaxy counts and luminosities, combined with a reasonable mass-to-light ratio, allow an estimate of the mass in cluster galaxies $M_{\text {gal }}$. With these three quantities, one can estimate the baryonic mass fraction of the cluster

$$
f_{b}=\frac{M_{\mathrm{gal}}+M_{\mathrm{ICM}}}{M_{\mathrm{tot}}} .
$$

In the absence of dissipation, one would expect the baryon fraction in the cluster to be representative of the baryon fraction of the universe; the baryons and dark matter in a cluster fell in from roughly the same comoving volume. We then have

$$
f_{b}=\frac{\rho_{b}}{\rho_{0}}=\frac{\Omega_{b}}{\Omega_{0}} .
$$

Cluster mass estimates give us $f_{b}$ and big bang nucleosynthesis theory gives limits on $\Omega_{b}$. The combination yields a prediction for the cosmological density parameter $\Omega_{0}$.

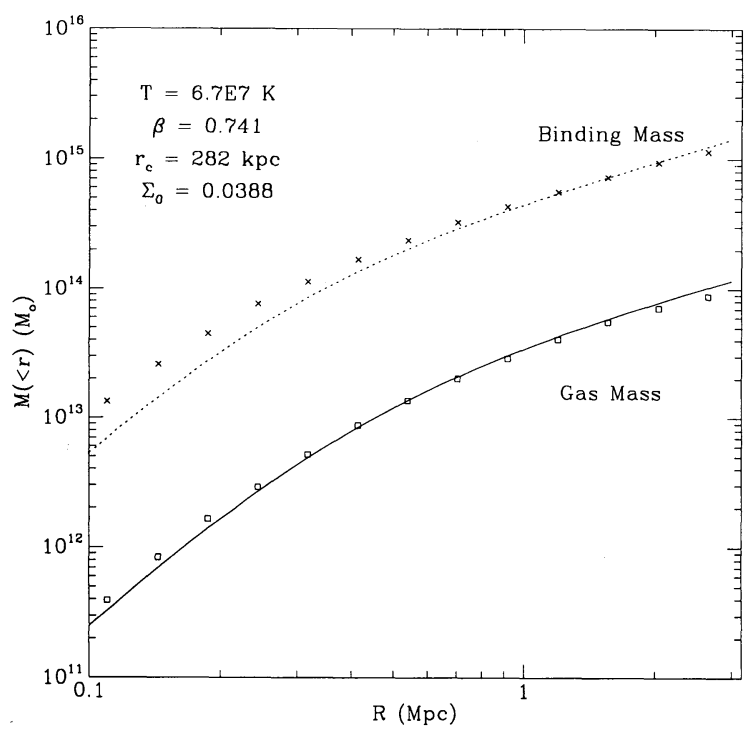

FIG. 12.-Binding and gas mass within radius (crosses and open squares), along with projections of hydrostatic isothermal $\beta$-model based on X-ray data, for the ejection run at a redshift $z=0.02$. X-ray surface brightness fitting parameters used are from the fit to the $z$-axis projection at that redshift. Temperature is the isothermal central temperature for that redshift from Fig. 2. Corrections for the softened force law used in the simulations are included. 


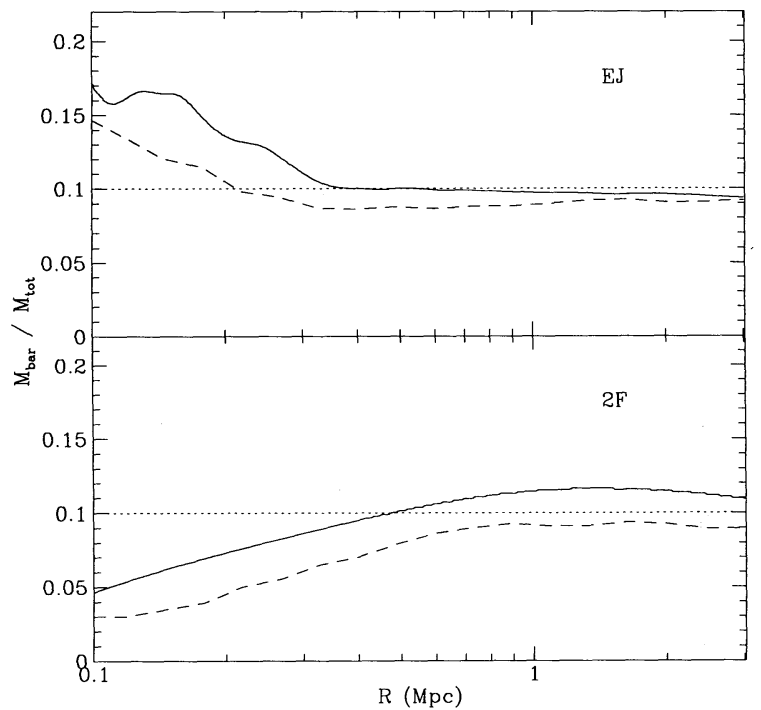

Fig. 13.-The baryon fraction of all matter within radius $r$, for the ejection and $2 \mathrm{~F}$ runs. The overall value of the baryon fraction for the simulations, 0.1 , is shown as a dotted line. The dashed lines indicate the true baryon fraction profiles, while the solid lines represent predictions for each simulation based on mass estimates from the hydrostatic isothermal $\beta$ model.

It is interesting to ask what baryon fraction would be inferred from "observing" the simulated cluster. Figure 13 shows the true baryon fraction as a function of radius for the runs with and without ejection. Also shown are an observer's prediction for this function; $M_{\mathrm{ICM}}$ and $M_{\text {tot }}$ are taken from the hydrostatic isothermal $\beta$-model mass estimates shown earlier, while $M_{\text {gal }}$ is determined by assuming that the observer knows perfectly the three-space distribution of galaxies (not just the projected distribution) as well as their actual masses. The true value of the baryon fraction for the simulation, $f_{\mathrm{b}}=0.1$, is shown as a dotted line across the plots. The central baryon fraction is above the global value in the ejection run because of the introduction of galaxies; baryons that may not have made it deep into the cluster in the $2 \mathrm{~F}$ run are carried in via the collisionless galaxy particles. The predicted value generally lies above the true value at low radii because the hydrostatic mass estimator is underestimating the binding mass at these radii, as shown in Figure 12. Beyond the central region, the predicted baryon fraction for both runs is never off by more than $50 \%$ from the true value. At $1 \mathrm{Mpc}$, the predicted value in both runs is similar to the global value; vigorous winds do not seem to dramatically affect the accuracy of estimates of the baryon fraction.

The best value of $\Omega_{b}$, from comparison of nucleosynthesis calculations with observed elemental abundances, is $\Omega_{b} h_{50}^{2}=$ $0.05 \pm 0.01$ (Walker et al. 1991). Meanwhile, observations of rich clusters suggest baryon fractions of $15 \%-30 \%$ (e.g., Briel, Henry, \& Böhringer 1992; David et al. 1992b). The two can be reconciled if the universe is open, $\Omega_{0}=0.15-0.3$. Analysis of our simulated cluster, which has an X-ray and optical appearance fairly typical of observed clusters, indicates that observational estimates of the baryon fraction are accurate to within $\sim 50 \%$. Reconciling the cluster baryon fraction with $\Omega=1$ is not possible solely by appealing to large systematic errors in mass estimates.

\subsection{Velocity Bias and the $\beta$-Problem}

Although they reside in a common potential well, the specific energies of the different mass components of the cluster-dark matter, galaxies, and gas-need not be exactly equal. Energy can be transferred among components; for example, by dynamical friction of dark matter acting on galaxies, or, for the gas, energy can be stored in either thermal or kinetic form, the latter mainly in bulk motions of the gas as it attempts to settle to equilibrium in a time-dependent potential well. Following the $\beta$-model convention, we define two values of $\beta$ based on the velocity dispersions of the dark matter and galaxies $\beta_{\mathrm{DM}} \equiv$ $\sigma_{\mathrm{DM}}^{2} /\left(k T / \mu m_{p}\right)$ and $\beta_{\mathrm{gal}} \equiv \sigma_{\mathrm{gal}}^{2} /\left(k T / \mu m_{p}\right)$. We use for $T$ a massweighted measure of the gas temperature. Because of the slightly negative radial temperature gradient, flux weighted temperatures are higher by $3 \%$ and $5 \%$ in the EJ and $2 \mathrm{~F}$ runs, respectively, consistent with the results of Navarro et al. (1993). We also define a "velocity bias" parameter (Carlberg 1991) as the ratio of velocity dispersions in galaxies and dark matter $b_{v} \equiv \sigma_{\mathrm{gal}} / \sigma_{\mathrm{DM}}$. All quantities are quoted within a comoving $1 \mathrm{Mpc}$ radius from the cluster center.

Figure 14 shows the evolution with redshift of $\beta$ for each species and $b_{v}$. No projection effects are considered; the temperature used is the true mass-averaged gas temperature over this volume, while the velocity dispersions used are $1 / \sqrt{3}$ of the true three-dimensional velocity dispersions, calculated from the particle distributions. The plot seems to show a periodicity, but this is deceptive; when time is used as the abscissa, no periodicity emerges. The source of the jumps in the value of $\beta$ for the galaxies is the low number statistics involved; no more than 30 or so galaxies are within the comoving $1 \mathrm{Mpc}$ radius at any time, and three galaxies infalling into the region can sharply change the velocity dispersion. After $z=0.4$, the value of $\beta_{\text {gal }}$ lies in the range $0.5-1.1$, with a mean and variance during that period of 0.74 and 0.15 , respectively. Almost identical mean and variance, 0.73 and 0.14 , are found in the run with truncated ejection.

At these lower redshifts, the values of $\beta_{\mathrm{DM}}$ lie in the range $0.9-1.1$, demonstrating that the ICM gas is able to thermalize rather efficiently. Still, there is a small amount $(\sim 10 \%-15 \%)$ of

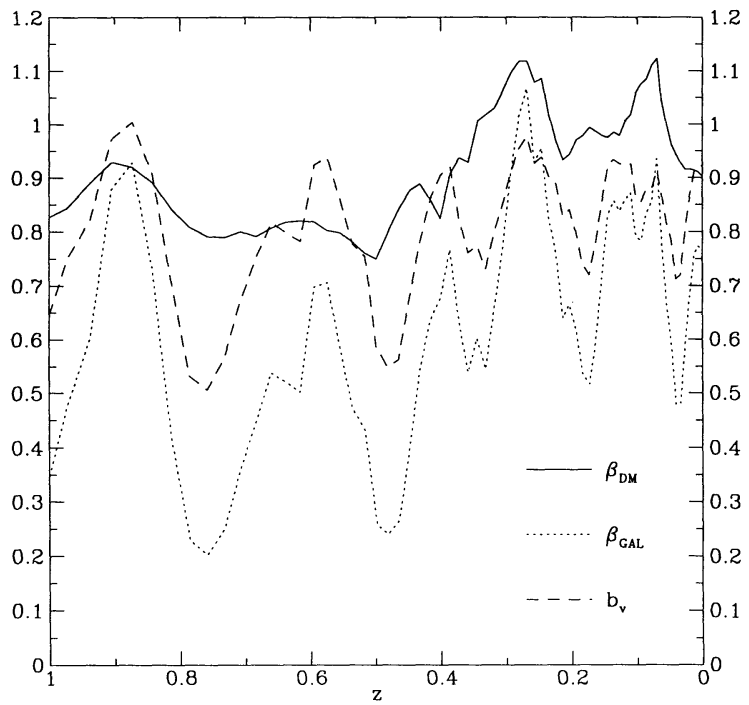

Fig. 14.-Redshift evolution of the true values of $\beta$, calculated using the mean temperature and one-dimensional velocity dispersion within a comoving radius of $1 \mathrm{Mpc}$. The solid line shows $\beta_{\mathrm{DM}}$ using the dark matter's velocity dispersion; the dotted line shows $\beta_{\mathrm{gal}}$ with a one-dimensional velocity dispersion calculated from the galaxies within $1 \mathrm{Mpc}$. The dashed line shows the velocity bias factor, expressed as the ratio of the two velocity dispersions, $b_{v}=\sigma_{\mathrm{gal}} / \sigma_{\mathrm{DM}}$. Some values from this plot are shown in Table 5. A persistent velocity bias is evident which leads to $\beta_{\text {gal }}<1$. 


\begin{tabular}{cccc}
\multicolumn{5}{c}{ TABLE 5 } \\
\multicolumn{4}{c}{$\beta_{\text {spec }}$ VALUES } \\
\hline \hline Redshift $z$ & Parameter & EJ & 2 F \\
\hline $0.25 \ldots \ldots$ & $\beta_{\text {DM }}$ & 1.09 & 1.20 \\
& $\beta_{\text {gal }}$ & 0.96 & $\ldots$ \\
$0.10 \ldots \ldots$ & $\beta_{\text {DM }}$ & 1.06 & 1.25 \\
& $\beta_{\text {gal }}$ & 0.79 & $\ldots$ \\
$0.06 \ldots \ldots$ & $\beta_{\text {DM }}$ & 1.05 & 1.06 \\
& $\beta_{\text {gal }}$ & 0.79 & $\ldots$ \\
$0.02 \ldots \ldots$ & $\beta_{\text {DM }}$ & 0.92 & 1.14 \\
& $\beta_{\text {gal }}$ & 0.68 & $\ldots$ \\
\hline
\end{tabular}

energy in kinetic form. This "loss" is compensated by the energy input from ejection. As evidence, the values of $\beta_{\mathrm{DM}}$ inferred from the $2 \mathrm{~F}$ run are typically $\sim 15 \%$ above the values in EJ run. These values are consistent with values of $\beta_{\mathrm{DM}}$ from other two-fluid simulations (Evrard 1990a, b). The mean and variance since $z=0.4$ are 1.00 and 0.07 for the EJ run and 1.17 and 0.06 for the $2 \mathrm{~F}$ model. The true values of $\beta$ for each species in the ejection run, as well as for the dark matter in the twofluid run, measured within a $1 \mathrm{Mpc}$ comoving radius from the center of the mass of the cluster, are shown in Table 5.

For the EJ run, "observed" values of $\beta_{\text {spec }}$, using the line-ofsight velocity dispersion from all galaxies within a projected Abell radius and an emission-weighted central temperature determined from a mock EXOSAT observation (2-10 keV, 45' FWHM), are $0.41,1.03$, and 0.48 for views along the three principal axes.

The data show a persistent velocity bias. The ratio of velocity dispersions $b_{v}$ climbs to 1 only once, at very high redshift; most of the time is spent at values between 0.8-0.9. The time average value since $z=0.4$ is $\left\langle b_{v}\right\rangle=0.86$ with a variance of 0.07 . These values imply a modest bias, consistent with results from two-fluid simulations modeling galaxy formation explicitly (Evrard, Summers, \& Davis 1994).

The presence of velocity bias conflicts with the conclusions of Lubin \& Bahcall (1993), who claim no evidence for velocity bias in a sample of 41 clusters with both measured X-ray temperatures and velocity dispersions based on 20 or more redshifts per cluster. The sample is largely that of David et al. (1993), who compiled temperatures based on the Einstein Monitor Proportional Counter (MPC), supplemented with data from Ginga, EXOSAT and HEAO 1 along with cluster velocity dispersions from the compilation of Struble \& Rood (1991). Lubin \& Bahcall (1993) claimed the data were well fitted by a scaling relation $\sigma \propto T^{0.5}$, implying a constant $\beta$ with best-fit value $\beta=0.94 \pm 0.08$. By assuming the $\mathrm{X}$-ray temperature was an unbiased estimator of the dark matter velocity dispersion (i.e., $\beta_{\mathrm{DM}} \equiv 1$ ), they directly connected $\beta$ to the velocity bias $\beta=b_{v}^{2}$. The $\beta$ determination then implies $b_{v}=$ $0.97 \pm 0.04$. Empirically, there seems to be no room for velocity bias.

However, there are some troubling aspects of this analysis. The values of $\beta$ span a large range, from 0.28 (A1142) to 3.13 (A1775), and the largest values tend to be the least accurately determined. Four clusters have values of $\beta$ which lie more than $3 \sigma$ away from the best fit value of 0.94 , and all lie below the best fit value. (The clusters are A85 $(\beta=0.55 \pm 0.10)$, A754 $(\beta=0.51 \pm 0.12), \mathrm{A} 1060(\beta=0.57 \pm 0.12)$, and $\bar{A} 1656$ (Coma; $\beta=0.56 \pm 0.10)$.) On the other hand, there are no clusters which lie more than $3 \sigma$ away from $\beta=0.74$, the time-averaged value from our simulation, which includes a modest velocity bias. Viewed this way, the observational data could be interpreted as actually favoring the presence of velocity bias.

\section{SUMMARY}

We have begun investigation of the effects of feedback due to winds from early-type galaxies on the dynamic and thermal history of intracluster medium. As a first analysis, we use an extreme ejection history, with the intent of clearly illustrating the direction in which specific cluster properties change. Compared to an otherwise identical model without ejection (a pure infall model), we find feedback affects the structure of the X-ray emitting gas in a Coma-sized cluster in the following ways.

1. At early times, feedback raises the entropy of protocluster gas, preventing it from being compressed to densities as high as those achieved in the infall case. Later gas settling onto higher adiabats is less affected. This results in both lower X-ray luminosities and more rapid X-ray evolution in the model with feedback compared to the infall case. Statistically complete surveys extending to $z \simeq 1$ would provide constraining power on the amount of feedback in clusters, although in a cosmologically dependent fashion. There is evidence from $R O S A T$ observations of an optically selected, high-redshift sample that Coma-sized clusters were much dimmer in X-rays at $z \simeq 1$ (Castander et al. 1993), but the small sample size (five clusters) and uncertainties due to projection effects on optical selection at high redshift hinder serious analysis. Clusters from the NEP region of the ROSAT all-sky survey may prove useful; empirical models suggest that $\sim 30$ clusters with $z>0.4(\sim 10 \%$ of the total) should be found (Evrard \& Henry 1991).

2. The ratio of specific energies in galaxies and gas, the $\beta$ parameter, is less than one in the ejection model. This differs from the infall case where $\beta>1$ is generic, as emphasized by Evrard (1990a, b). The different behavior arises from the combined effects of a slightly higher gas temperature (due to energy input from the galactic winds) and a velocity bias for the galaxy population. For our Coma-sized model, the temperature increase is $\sim 15 \%$ over the infall case and the ratio of galaxy to dark matter velocity dispersions (the velocity bias) is typically 0.85 . Since the infall models do not explicitly model galaxies, an assumption of no velocity bias is usually made, leading to estimates of $\beta \simeq 1.2$. Incomplete thermalization of the gas and poor (at the $\sim 15 \%$ level) modeling of the dark matter distribution are responsible for $\beta>1$ in this case (Evrard 1990b). We favor the ejection model predictions over the infall case because of the improvement in physical modeling represented by explicitly including the galaxy population. We note that several simulations using independent methods have produced a value of the velocity bias similar in magnitude to that seen in our ejection experiment (see Evrard et al. 1994 and references therein). Interpretation of observational data remains problematic. Edge \& Stewart (1991) favored spectroscopic values of $\beta<1$, but noted that $\beta>1$ was common for the richest clusters. Lubin \& Bahcall (1993) claimed the data are consistent with a constant value of $\beta$ very close to unity, although individual values span a range from 0.28 to 3.1. A combination of multifiber optical spectroscopy and spatially resolved X-ray spectroscopy with the $A S C A$ satellite on a statistical sample of clusters would go a long way toward settling this matter.

3. The morphology of X-ray emission is little affected by feedback; both the ejection and infall runs produce clusters 
with moderately elliptic contours well fitted by the standard form (eq. [8]) with values of $\beta_{\mathrm{fit}}$ in the range $0.7-1$. There is a tendency for lower $\beta_{\mathrm{fit}}$ values in the ejection run, as might be expected from energetic arguments. However, this difference is small compared to variations in $\beta_{\mathrm{fit}}$ caused by dynamics. Mergers are common and the relaxation time in the outer parts of the cluster is sufficiently long that the density structure at large radii ( $r \gtrsim 1 \mathrm{Mpc}$ ) generally deviates from its hydrostatic equilibrium expectation. One should not expect tight agreement between $\beta_{\mathrm{fit}}$ and $\beta_{\mathrm{gal}}$ in any model in which cluster formation involves a large degree of recent merging.

4. Estimates of the gas and binding mass profiles of our simulated clusters, based upon X-ray observations and subsequent application of the $\beta$-model and hydrostatic equilibrium, are accurate to better than $20 \%$ for both the EJ and $2 \mathrm{~F}$ runs at radii beyond $500 \mathrm{kpc}$. The inclusion of energetic winds does not seem to affect the state of cluster gas strongly enough to detrimentally affect the quality of such mass estimates. Dynamical mass estimates based on galaxy line-of-sight velocity dispersions, on the other hand, showed errors of as much as factors of 2-3.

5. Heavy element enrichment by winds produces a radially decreasing abundance gradient. The average iron abundance within $300 \mathrm{kpc}$ is more than half the abundance in the galactic ejecta, dropping below one-sixth this value at radii beyond 2 $\mathrm{Mpc}$. The gradient is due to the steeper radial profile of galaxy number density with respect to intracluster gas. Observations of a central concentration of iron do not demand that ram pressure stripping was the mechanism for its deposition. The metal gradient showed no sign of weakening during a modest merging encounter. Observed with a nonimaging, spectroscopic satellite similar to EXOSAT or HEAO 1, the gradient becomes extremely difficult to detect, due to contamination by core emission in off-center pointings. Arcminute resolution spectroscopy, such as is available on ASCA, would uncover the radial gradient. Depending on the degree of mixing, the observations may also reveal small-scale structure in the iron abundance (see Fig. 8).

There is much work yet to be done before we can claim a true understanding of the origin and history of the ICM and its metal content. The work presented in this paper represents a first essay at modeling the effect of galactic winds in three dimensions in a cosmologically viable scenario. The nature of the problem is such that there is, unavoidably, a moderately large parameter space associated with the modeling which embodies the present uncertainties in galaxy formation and in modelling winds from evolving galaxies.

We plan to explore the sensitivity of the results above to parameters controlling the wind modeling (ejection rates, luminosities, wind metallicity, etc.), specifically for ejection models which are based on our current understanding of the evolution of early-type galaxies. For a fixed feedback model, we will study a set of clusters spanning a reasonable range of richness, in order to examine the effect of feedback on correlations of $\mathrm{X}$-ray and optical properties. We also intend to study models in which gas-dynamical stripping is the dominant source of cluster metals.

C. A. M. would like to thank Hans Böhringer, Joel Bregman, Larry David, Rick Mushotzky, Alvio Renzini, and Doug Richstone for very educational discussions. This work was supported by NASA Theory Grant NAGW-2367. C. A. M. also acknowledges support from a Rackham Predoctoral Fellowship and a Sokol International Research Fellowship.

\section{APPENDIX}

The emissivity due to free-free encounters between electrons and ions in a fully ionized primordial plasma $(X=0.76, Y=0.24)$ with temperature $T$ and particle density $n$ is given by (Spitzer 1968)

$$
\epsilon_{v}=4.32 \times 10^{-28} n^{2} T^{1 / 2}(h / k T) \bar{g}(h v / k T) e^{-h v / k T} \operatorname{ergs~s}^{-1} \mathrm{~cm}^{-3} \mathrm{~Hz}^{-1}
$$

where $\bar{g}(h v / k T)$ is an averaged Gaunt factor providing quantum mechanical corrections to the classical expression. Define a bolometric emissivity using unit Gaunt factor

$$
\epsilon_{\mathrm{bol}}=\int_{0}^{\infty} d v \epsilon_{v}=4.32 \times 10^{-28} n^{2} T^{1 / 2} \operatorname{ergs~s}^{-1} \mathrm{~cm}^{-3} .
$$

Then the energy radiated within a given energy band $E_{1}-E_{2}$ can be expressed as

$$
\epsilon_{\text {band }}=f_{\text {band }}(T) \epsilon_{\text {bol }} \text {, }
$$

where

$$
f_{\text {band }}(T)=\int_{E_{1} / k T}^{E_{2} / k T} d \eta \bar{g}(\eta) e^{-\eta}
$$

The band limited $\mathrm{X}$-ray emission from a given volume $V$ is found by

$$
L_{X}=\int_{V} d^{3} x \epsilon_{\text {band }}(x)=4.32 \times 10^{-28} \int_{V} d^{3} x n^{2}(x) T^{1 / 2}(x) f_{\text {band }}[T(x)] \text { ergs s }^{-1} \text {. }
$$
Using the discrete information in the simulations, $\int_{V} d^{3} x \rho \rightarrow \sum_{i} m_{i}$, implies the X-ray luminosity from a given volume can be
calculated by

$$
L_{X}=4.32 \times 10^{-28}\left(\mu m_{p}\right)^{-2} m_{g} \sum_{i} \rho_{i} T_{i}^{1 / 2} f_{\text {band }}\left(T_{i}\right) \text { ergs s }^{-1}
$$


where the sum runs over particles within the volume. The flux $F_{x}$ of X-rays at Earth in an observed energy band $E_{1}-E_{2}$ is (Weinberg 1972)

$$
F_{X}=(4 \pi)^{-1}\left(\frac{H_{0}}{2 c}\right)^{2}\left[\frac{L_{\text {bol }} f_{\text {band }}(T, z)}{(1+z)^{2}\left(1-(1+z)^{-1 / 2}\right)^{2}}\right],
$$

where $f_{\text {band }}(T, z)$ is the integral given by equation (A4) over an appropriately redshifted energy range $E_{1}(1+z)$ to $E_{2}(1+z)$.

\section{REFERENCES}

Bardeen, J. M., Bond, J. R., Kaiser, N., \& Szalay, S. 1986, ApJ, 304, 15

Bertschinger, E. 1987, ApJ, 323, L103

Biermann, P. 1978, A\&A, 62, 255

Binney, J., \& Tremaine, S. 1987, Galactic Dynamics (Princeton: Princeton University Press)

Blumenthal, G. R., Faber, S. M., Primack, J. R., \& Rees, M. J. 1984, Nature, 311,517

Briel, U. G., Henry, J. P., \& Böhringer, H. 1992, A\&A, 259, L31

Carlberg, R. G. 1991, ApJ, 367, 385

Carr, B. J., Bond, J. R., \& Arnett, W. D. 1984, ApJ, 277, 445

Castander, F. J., Ellis, R. S., Frenk, C. S., Dressler, A., \& Gunn, J. E. 1993, ApJ, 424, 79

Cavaliere, A., \& Fusco-Femiano, R. 1976, A\&A, 49, 137

1978, A\&A, 70, 677

Ciotti, L., D’Ercole, A., Pellegrini, S., \& Renzini, A. 1991, ApJ, 376, 380

Cowie, L. L., \& Perrenod, S. C. 1978, ApJ, 219, 354

Crone M. M. Evrard, A. E \& Richstone, D. O. 1994, ApJ, 434, 402

David, L. P., Arnaud, K. A., Forman, W., \& Jones, C. 1990a, ApJ, 356, 32

David, L. P., Forman, W., \& Jones, C. 1990b, ApJ, 359, 29 1991a, ApJ, 369, 121

- 1991b, ApJ, 380, 39

David, L. P., Hughes, J. P., \& Tucker, W. H. 1992a, ApJ, 394, 452

David, L. P., Jones, C., \& Forman, W. 1992b, BAAS, 24, 1145

Davis, M., Efstathiou, G., Frenk, C. S., \& White, S. D. M. 1985, ApJ, 292, 371

Dressler, A. 1980, ApJ, 341, 26

Dubinski, J., \& Carlberg, R. G. 1991, ApJ, 378, 496

Edge, A. C., \& Stewart, G. C. 1991, MNRAS, 252, 428

Efstathiou, G., Davis, M., Frenk, C. S., \& White, S. D. M. 1985, ApJ Suppl., 57, 241

Efstathiou, G., \& Eastwood, J. W. 1981, MNRAS, 194, 503

Evrard, A. E. 1988, MNRAS, 235, 911

. 1990a, Clusters of Galaxies, ed. W. Oegerle, M. Fitchett, \& L. Danly (Cambridge: Cambridge Univ. Press), 287

1990b, ApJ, 363, 349

Evrard, A. E., \& Henry, J. P. 1991, ApJ, 383, 95

Evrard, A. E. Metzler, C. A \& Navarro, J. F. 1994, in preparation

Evrard, A. E., Silk, J., \& Szalay, A. S. 1990, ApJ, 365, 13

Evrard, A. E., Summers, F. J., \& Davis, M. 1994, ApJ, 422, 11

Frenk, C. S., White, S. D. M., Davis, M., \& Efstathiou, G. 1988, ApJ, 327, 507

Gaetz, T. J., Salpeter, E. E., \& Shaviv, G. 1987, ApJ, 316, 530

Gingold, R. A., \& Monaghan, J. J. 1977, MNRAS, 181, 375

Gull, S. F., \& Nothover, K. J. 1975, MNRAS, 173, 585

Gunn, J. E., \& Gott, J. R. 1972, ApJ, 176, 1

Heckman, T. M., Armus, L., \& Miley, G. K. 1987, AJ, 93, 276

1990, ApJS, 74, 833

Hockney, R. W., \& Eastwood, J. W. 1981, Computer Simulation Using Particles (New York: McGraw Hill)

Hughes, J. P., Butcher, J. A., Stewart, G. C., \& Tanaka, Y. 1992, ApJ, 404, 611

Hughes, J. P., Gorenstein, P., \& Fabricant, D. 1988, ApJ, 329, 82

Jones, C., \& Forman, W. 1992, in Clusters and Superclusters of Galaxies, ed. A. C. Fabian (Dordrecht: Kluwer), 49
Just, A., Deiss, B. M., Kegel, W. H., Bohringer, H., \& Morfill, G. E. 1990, ApJ, 354,400

Kaiser, N. 1991, ApJ, 383, 104

Katz, N., Quinn, T., \& Gelb, J. 1993, MNRAS, 265, 689

Katz, N., \& White, S. D. M. 1993, ApJ, 412, 455

Kowalski, M. P., Cruddace, R. G., Snyder, W. A., Fritz, G. G., Ulmer, M. P., \& Fenimore, E. E. 1993, ApJ, 412, 489

Larson, R. B., \& Dinerstein, H. L. 1975, PASP, 87, 911

Lea, S. M. 1976, ApJ, 203, 569

Lea, S. M., \& Holman, G. D. 1978, ApJ, 222, 29

Loveday, J., Peterson, B. A., Efstathiou, G., \& Maddox, S. J. 1992, ApJ, 390, 338

Lubin, L. M., \& Bahcall, N. A. 1993, ApJ, 415, 17

Matteucci, F, \& Tornambè, A. 1987, A\&A, 185, 51

Matteucci, F., \& Vettolani, G. 1988, A\&A, 202, 21

McCarthy, P., Heckman, T., \& van Bruegel, W. 1987, AJ, 93, 264

Monaghan, J. J. 1992, ARA\&A, 30, 543

Mushotzky, R. F. 1991, in Clusters and Superclusters of Galaxies, ed. A. C. Fabian (Dordrecht: Kluwer), 91

A. C. Fabian (1994, in Clusters of Galaxies, ed. J. Trân Thanh Vân, F. Durret, A. Mazure, \& S. White, in press

Navarro, J. F., Frenk, C. S., \& White, S. D. M. 1994, MNRAS, submitted

Perrenod, S. C. 1978, ApJ, 226, 566

Ponman, T. J., Bertram, D., Church, M. J., Eyles, C. J., Skinner, G. K., Watt, M. P., \& Willmore, A. P. 1990, Nature, 347, 450

Renzini, A., Ciotti, L., D'Ercole, A., \& Pellegrini, S. 1993, ApJ, 419, 52

Rephaeli, Y. 1977, ApJ, 218, 323

1979, ApJ, 227,364

Rephaeli, Y., \& Salpeter, E. E. 1980, ApJ, 240, 20

Roettiger, K., Burns, J., \& Loken, C. 1993, ApJ, 407, 53

ROSAT Scientific Data Center, 1989, ROSAT Mission Description, NRA 89-OSSA-2, 10.3.

Sarazin, C. L. 1986, Rev. Mod. Phys., 58, 1

Sarazin, C. L., \& Bahcall, J. N. 1977, ApJS, 34, 451

Struble, M. F., \& Rood, H. J. 1991, ApJS, 77, 363

Takeda, H., Nulsen, P. E. J., \& Fabian, A. C. 1984, MNRAS, 208, 261

Thomas, P. A., \& Couchman, H. M. P. 1992, MNRAS, 257, 11

Tsai, J. C. Katz N. \& Bertschinger, E. 1994, ApJ, 423, 553

Walker, T. P., Steigman, G., Schramm, D. N., Olive, K. A., \& Kang, H. 1991, ApJ, 376, 51

Watt, M. P., Ponman, T. J., Bertram, D., Eyles, C. J., Skinner, G. K., \& Willmore, A. P. 1992, MNRAS, 258, 738

Weinberg, S. 1972, Gravitation and Cosmology (New York: Wiley)

White, R. E. 1991, ApJ, 367, 69

White, S. D. M. 1976, MNRAS, 177, 717

. 1980, MNRAS, 191, 1P

White, S. D. M., Navarro, J. F., Evrard, A. E., \& Frenk, C. S. 1993, Nature, 366, 429

Yahil, A., \& Ostriker, J. P. 1973, ApJ, 185, 787 